\title{
OPEN Development of short and long-range magnetic order in the double perovskite based frustrated triangular lattice antiferromagnet $\mathrm{Ba}_{2} \mathrm{MnTeO}_{6}$
}

\author{
J. Khatua ${ }^{1,7,8}$, T. Arh ${ }^{2,3}$, Shashi B. Mishra ${ }^{4}$, H. Luetkens ${ }^{5}$, A. Zorko ${ }^{2,3 凶}$, B. Sana ${ }^{1}$, \\ M. S. Ramachandra Rao $0^{6,7}$, B. R. K. Nanda ${ }^{4,8,9 凶}$ \& P. Khuntia ${ }^{1,7,8}$
}

Frustrated magnets based on oxide double perovskites offer a viable ground wherein competing magnetic interactions, macroscopic ground state degeneracy and complex interplay between emergent degrees of freedom can lead to correlated quantum phenomena with exotic excitations highly relevant for potential technological applications. By local-probe muon spin relaxation ( $\mu \mathrm{SR})$ and complementary thermodynamic measurements accompanied by first-principles calculations, we here demonstrate novel electronic structure and magnetic phases of $\mathrm{Ba}_{2} \mathrm{MnTeO}_{6}$, where $\mathrm{Mn}^{2+}$ ions with $\mathrm{S}=$ 5/2 spins constitute a perfect triangular lattice. Magnetization results evidence the presence of strong antiferromagnetic interactions between $\mathrm{Mn}^{2+}$ spins and a phase transition at $T_{N}=20 \mathrm{~K}$. Below $T_{N}$, the specific heat data show antiferromagnetic magnon excitations with a gap of $1.4 \mathrm{~K}$, which is due to magnetic anisotropy. $\mu$ SR reveals the presence of static internal fields in the ordered state and shortrange spin correlations high above $T_{N}$. It further unveils critical slowing-down of spin dynamics at $T_{N}$ and the persistence of spin dynamics even in the magnetically ordered state. Theoretical studies infer that Heisenberg interactions govern the inter- and intra-layer spin-frustration in this compound. Our results establish that the combined effect of a weak third-nearest-neighbour ferromagnetic inter-layer interaction (owing to double-exchange) and intra-layer interactions stabilizes a three-dimensional magnetic ordering in this frustrated magnet.

Incompatibility of magnetic interactions with a spin lattice leads to spin frustration and strong quantum fluctuations yielding novel states in quantum materials ${ }^{1-5}$. Triangular lattice antiferromagnet offers the simplest prototype to realize frustration induced ground states. In this antiferromagnet, the complex interplay between several degrees of freedom and strong quantum fluctuations leads to various types of intriguing physical phenomena such as quantum spin liquids ${ }^{6,7}$, field induced magnetization plateaus ${ }^{8-10}$, non-colinear $120^{\circ}$ magnetic ordered states $^{11}$, spin-driven ferroelectricity ${ }^{12,13}$, etc. Experimentally, it has been shown that the triangular lattice with low spin is ideal to realize exotic spin-liquid ground state due to enhanced zero-point spin fluctuations as observed, e.g., in $k$-(BEDT-TTF $)_{2} \mathrm{Cu}_{2}(\mathrm{CN})_{3}{ }^{14,15}$, EtMe $3 \mathrm{Sb}\left[\mathrm{Pd}(\mathrm{dmit})_{2}\right]_{2}{ }^{16,17}$ and $1 \mathrm{~T}-\mathrm{TaS}_{2}{ }^{18}$. On the other hand, for classical Heisenberg spins on triangular lattices, inter-planar interaction, next-nearest-neighbor interaction and anisotropic interaction suppress low dimensional magnetism resulting in three-dimensional $(3 D)$ magnetic

\footnotetext{
${ }^{1}$ Department of Physics, Indian Institute of Technology Madras, Chennai 600036, India. ${ }^{2}$ Jožef Stefan Institute, Jamova c. 39, 1000 Ljubljana, Slovenia. ${ }^{3}$ Faculty of Mathematics and Physics, University of Ljubljana, Jadranska u. 19, 1000 Ljubljana, Slovenia. ${ }^{4}$ Condensed Matter Theory and Computational Lab, Department of Physics, Indian Institute of Technology Madras, Chennai 600036, India. ${ }^{5}$ Laboratory for Muon-Spin Spectroscopy, Paul Scherrer Institute, $\mathrm{CH}-5232$ Villigen, Switzerland. ${ }^{6}$ Department of Physics, Nano Functional Materials Technology Centre and Materials Science Research Centre, Indian Institute of Technology Madras, Chennai 600036, India. ${ }^{7}$ Quantum Centre for Diamond and Emergent Materials, Indian Institute of Technology Madras, Chennai 600036, India. ${ }^{8}$ Functional Oxide Research Group, Indian Institute of Technology Madras, Chennai 600036, India. ${ }^{9}$ Atomistic Modelling and Materials Design Group, Indian Institute of Technology Madras, Chennai 600036, India. ${ }^{\boxplus}$ email: andrej.zorko@ijs.si; nandab@iitm.ac.in; pkhuntia@iitm.ac.in
} 
ordered states as observed in various compounds, e.g., $\mathrm{RbFe}\left(\mathrm{MoO}_{4}\right)_{2}\left(\mathrm{Fe}^{3+}, S=5 / 2\right)^{19}, \mathrm{NaBa}_{2} \mathrm{Mn}_{3} \mathrm{~F}_{11}\left(\mathrm{Mn}^{2+}, S\right.$ $=5 / 2)^{20}, \mathrm{LiCrO}_{2}\left(\mathrm{Cr}^{3+}, S=3 / 2\right)^{21}, \mathrm{Rb}_{4} \mathrm{Mn}\left(\mathrm{MoO}_{4}\right)_{3}\left(\mathrm{Mn}^{2+}, S=5 / 2\right)^{22,23}, \mathrm{Ba}_{8} \mathrm{MnNb}_{6} \mathrm{O}_{24}\left(\mathrm{Mn}^{2+}, S=5 / 2\right)^{24}$, etc. In such classical spin systems strong thermal fluctuations lead to magnetically ordered ground state via "order by disorder" mechanism ${ }^{25}$. The physics of frustrated triangular lattice is rich and diverse $\mathrm{e}^{1,26,27}$, however, in many cases anti-site disorder, anisotropy and inter-plane interactions put a strong constraint on the ground-state spin dynamics and challenge theoretical paradigms ${ }^{28-31}$. The current challenge is to explore disorder-free frustrated triangular lattice antiferromagnets with exactly solvable ground state with potential to host exotic magnetism and spin dynamics.

In this context, $B$ site ordered double perovskites of general formula $A_{2} B B^{\prime} \mathrm{O}_{6}$, where $A$ represents a divalent cation, $B$ is a $3 d$ transition metal ion and $B^{\prime}=\mathrm{Te}^{6+}, \mathrm{Mo}^{6+}$ or $\mathrm{W}^{6+}$ offer an alternate route to realization of novel magnetism and spin dynamics as a result of intricate interplay between spin, lattice and charge degrees of freedom. It has been observed that many unconventional magnetic ground states are governed by planar structure of $B$-site ions. For example, $\mathrm{Ba}_{2} \mathrm{CoTeO}_{6}$ is a unique case of $\mathrm{B}$-site ordered double perovskite, where $\mathrm{Co}^{2+}(S=1 / 2)$ ions form two (triangular and honeycomb) subsystems. The spins on the triangular lattice behave as Heisenberg spins, while the spins on the honeycomb lattice show Ising like antiferromagnetic interactions ${ }^{32,33}$. Electronspin resonance (ESR) and magnetization measurements show that applied magnetic field perpendicular to the easy-axis induces magnetization plateaus for both sub-lattices due to strong quantum effects of $S=1 / 2 \operatorname{spins}^{32-34}$. Another interesting example is $\mathrm{Sr}_{2} \mathrm{CuTeO}_{6}$, a quasi-two dimensional Heisenberg antiferromagnet, where $\mathrm{Cu}^{2+}(S$ $=1 / 2$ ) ions form a planar square lattice and develop Néel order below $29 \mathrm{~K}^{35}$. Interestingly, magnetic susceptibility, specific heat and $\mu$ SR studies on $\mathrm{Sr}_{2} \mathrm{CuTe}_{1-x} \mathrm{~W}_{x} \mathrm{O}_{6}$ demonstrated a quantum disordered ground state for $x$ $=0.5^{36}$. This is a promising candidate to tune electron correlation by quenched disorder in the $J_{1}-J_{2}$ Heisenberg model on a square lattice.

Recently, a new B-site ordered double perovskite $\mathrm{Ba}_{2} \mathrm{MnTeO}_{6}$ (henceforth $\mathrm{BMTO}$ ), where $\mathrm{Mn}^{2+}$ ions with spin $S=5 / 2$ constitute a perfect spin lattice without anti-site disorder, has been reported ${ }^{37-39}$. While one of the study proposed a cubic space group $(\mathrm{Fm} \overline{3} \mathrm{~m})$, the other suggested a trigonal space group $\mathrm{R} \overline{3} \mathrm{~m}$ for describing the structure of $\mathrm{BMTO}^{37,38}$. The same trigonal space group was proposed for the BMTO also in an earlier study ${ }^{39}$. The high-temperature magnetic susceptibility data follow the Curie-Weiss law with large Curie-Weiss temperature, which suggests the presence of strong antiferromagnetic interaction between $\mathrm{Mn}^{2+}$ spins. An anomaly is observed in the magnetic susceptibility and specific heat data at $T_{N}=20 \mathrm{~K}$, which is an indication of a symmetry breaking phase transition in BMTO. However, a clear picture of the crystal structure and exchange interaction between $\mathrm{Mn}^{2+}$ spins of BMTO is missing. Also, the presence of static internal fields, the development of the order parameter and spin dynamics above and below the Néel temperature of this novel antiferromagnet have not been yet explored.

In this work, we report our results based on XRD, magnetization, specific heat, and muon spin relaxation $(\mu$ SR) studies as well as density functional theory (DFT) calculations in order to shed new insight into the crystal structure, magnetism, anisotropy and spin correlations in this novel frustrated triangular lattice antiferromagnet. We have found that the trigonal and cubic structures can both index the observed XRD peaks in BMTO, as also concluded previously ${ }^{38}$. All structural data thus indicate that the two structures are only marginally different, so that the exact structure likely has no significant effect on magnetism and spin dynamics. Indeed, it is suggested that the two structures are very close if one converts one space group to the other. As the trigonal space group offers an additional degree of freedom for the positions of $\mathrm{Ba}$ and $\mathrm{O}$ sites along the $c$-axis, this suggests that the trigonal space group may be advantageous over the cubic one $\mathrm{e}^{34,38,40,41}$. The Néel ordering at $T_{N}=20 \mathrm{~K}$ is confirmed by local-probe $\mu \mathrm{SR}$ measurements revealing the appearance of static internal fields below $T_{N}$. These measurements show that the whole sample enters a long-range magnetically ordered state below this temperature, while short-range correlations are observed all the way up to $35 \mathrm{~K}$. Moreover, $\mu \mathrm{SR}$ reveals critical slowing-down of spin dynamics at $T_{N}$ and its persistence to the lowest temperatures, as well as tracks the development of the order parameter. Furthermore, we find that a broad maximum at $10 \mathrm{~K}$ in the magnetic specific heat data can be associated with a magnon gap due to magnetic anisotropy. Namely, the magnetic specific heat data below $T_{N}$ reveals magnon excitations with a gap of $\sim 1.4 \mathrm{~K}$. The origin of magnetic ordering in BMTO is studied within the framework of density functional theory (DFT) calculations for the trigonal space group. Our results reveal that the $\mathrm{Mn}^{2+}$ spins favor a dominant Heisenberg antiferromagnetic ordering consistent with the experimental results. Our calculations using the DFT $+U$ formalism yield intra-layer exchange energy $J_{1}=4.6$ $\mathrm{K}$ and a comparable inter-layer exchange coupling $J_{2}=0.92 J_{1}$. In addition, a weaker ferromagnetic inter-layer interaction exists with third nearest neighbor $\left(J_{3}=-0.04 J_{1}\right)$ due to double-exchange interaction via the linear path $\mathrm{Mn}-\mathrm{O}-\mathrm{Te}-\mathrm{O}-\mathrm{Mn}$. Though the strength of this indirect interaction is more than an order of magnitude smaller than the leading AFM interaction, the combined effect of $J_{2}$ and $J_{3}$ contributes towards stabilizing the long-range magnetic order in this frustrated magnet.

\section{Results}

XRD and structural details. To check the phase purity, we measured the XRD pattern of polycrystalline BMTO samples. Figure 1c depicts the powder XRD pattern at room temperature. Rietveld refinement of XRD data using GSAS software ${ }^{43}$ reveals that BMTO crystallizes in trigonal crystal structure with the space group $\mathrm{R} \overline{3} \mathrm{~m}$ (No.166) and gives lattice parameters (Table 1) that are consistent with those previously reported ${ }^{38,39}$. Our analysis reveals the absence of any site disorder between constituent atoms in BMTO. In the BMTO crystal structure shown in Fig. $1 \mathrm{a} \mathrm{Mn}{ }^{2+}\left(3 d^{5} ; S=5 / 2\right)$ and $\mathrm{Te}^{6+}\left(4 d^{10} ; S=0\right)$ ions form $\mathrm{MnO}_{6}$ and $\mathrm{TeO}_{6}$ octahedra with nearest-neighbor oxygen ions, respectively. The $\mathrm{Mn}-\mathrm{O}$ bond length within the $\mathrm{MnO}_{6}$ octahedra is $2.179 \AA$ and the $\mathrm{Te}-\mathrm{O}$ bond length within the $\mathrm{TeO}_{6}$ is $1.932 \AA$. In the $a-b$ plane, the nearest-neighbor $(5.81 \AA) \mathrm{Mn}^{2+}$ ions form equilateral triangular planes stacked along the $c$ axis (see Fig. 1b). The consecutive $\mathrm{Mn}^{2+}$ triangular planes 

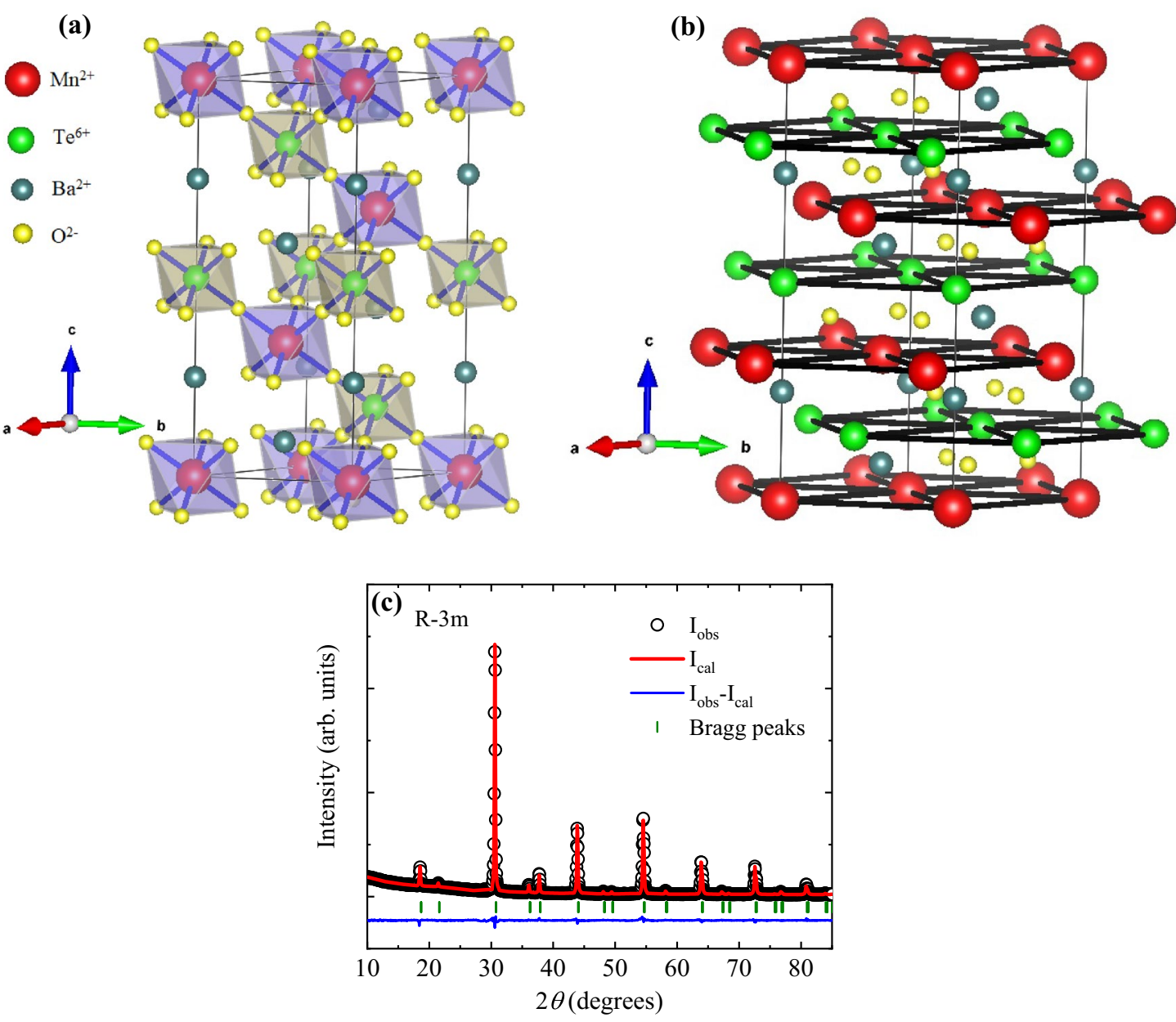

Figure 1. (a) The arrangement of $\mathrm{MnO}_{6}$ (purple) and $\mathrm{TeO}_{6}$ (dark yellow) octahedra in the unit cell of BMTO. (b) Schematic of consecutive triangular layers of $\mathrm{Mn}^{2+}$ ions (red) separated by triangular layer of $\mathrm{Te}^{6+}$ ions (green) along $c$ axis. The VESTA software ${ }^{42}$ was used for visualization of the crystal structure. (c) Rietveld refinement profile of X-ray diffraction data with the solid line $\left(I_{c a l}\right)$ through the experimental points $\left(I_{o b s}\right)$ calculated for trigonal crystal structure of BMTO. The olive vertical bars indicate the position of the Bragg reflections and the residual data are denoted by the blue solid line.

\begin{tabular}{|l|l|l|l|l|l|}
\hline Atom & Wyckoff position & $\mathbf{x}$ & $\mathbf{y}$ & $\mathbf{z}$ & Occ. \\
\hline $\mathrm{Mn}$ & $3 a$ & 0 & 0 & 0 & 1 \\
\hline $\mathrm{Te}$ & $3 b$ & 0 & 0 & 0.5 & 1 \\
\hline $\mathrm{Ba}$ & $6 c$ & 0 & 0 & 0.25 & 1 \\
\hline $\mathrm{O}$ & $18 h$ & 0.489 & 0.51 & 0.245 & 1 \\
\hline
\end{tabular}

Table 1. Rietveld refinement results at room temperature for BMTO with space group $R \overline{3} m$ and unit cell parameters $a=b=5.814 \AA, c=14.243 \AA$ and $\alpha=90^{\circ}, \beta=90^{\circ}, \gamma=120^{\circ}$. The goodness of Rietveld refinement is confirmed by the following factors: $\chi^{2}=4.8 ; \mathrm{R}_{w p}=6 \% ; \mathrm{R}_{\exp }=2.72 \%$ and $\mathrm{R}_{p}=4 \%$.

are separated by non-magnetic triangular planes of $\mathrm{Te}^{6+}$ (Fig. 1b). The adjacent inter-layer Mn-Mn distance is $5.81 \AA$. The nearest-neighbor $\mathrm{Mn}^{2+}$ ions of $\mathrm{MnO}_{6}$ octahedra are connected via $\mathrm{TeO}_{6}$ octahedra. The interplanar $\mathrm{Mn}^{2+}$ ions are connected through the linear path $\mathrm{Mn}^{2+}(1)-\mathrm{O}^{2-}-\mathrm{Te}^{6+}-\mathrm{O}^{2-}-\mathrm{Mn}^{2+}(2)$, here $\mathrm{Mn}^{2+}(1)$ and $\mathrm{Mn}^{2+}(2)$ refer to $\mathrm{Mn}^{2+}$ ions in two adjacent planes. We found a similarity between the structure of BMTO with the rare-earth based spin-liquid candidate $\mathrm{YbMgGaO}_{4}$, though the latter is composed of $4 f$ magnetic ions ${ }^{44}$. $\mathrm{YbMgGaO}_{4}$ crystallizes in the same space group $R \overline{3} m$ with lattice parameters $a=b=3.41 \AA$ and $c=25.14 \AA^{44}$. In $\mathrm{YbMgGaO}_{4}$, a single crystallographic site $(3 \mathrm{a})$ of $\mathrm{Yb}$ atoms and atomic coordinate $(0,0,0)$ matches with coordinate of $\mathrm{Mn}^{2+}$ in BMTO. Although both systems belong to the same crystal class, the spin-orbit coupling plays an important role to host an exotic ground state in $\mathrm{YbMgGaO}_{4}{ }^{45}$, whereas in BMTO the inherent physics 

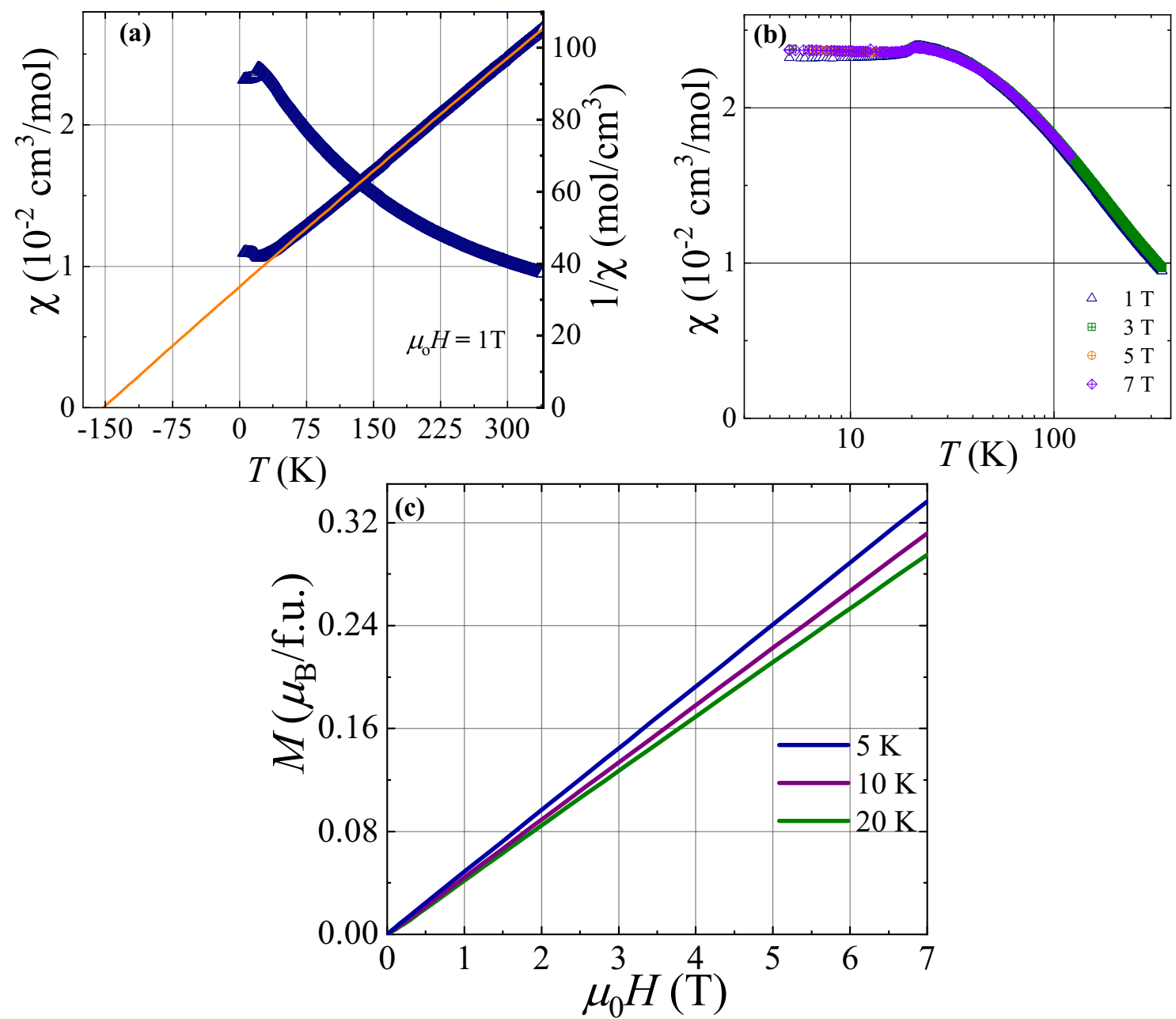

Figure 2. (a) The temperature dependence of dc magnetic susceptibility $(\chi(T)$; left axis) and inverse magnetic susceptibility $(1 / \chi(T)$; right axis ) with the Curie-Weiss fit. (b) The temperature dependence of $\chi(T)$ for different magnetic fields in the temperature range $5 \mathrm{~K} \leq T \leq 100 \mathrm{~K}$. (c) Magnetization versus field at several temperatures.

of high-spin-state of the magnetic ions is expected to be different due to much smaller spin-orbit coupling and the presence of finite inter-plane interactions.

Magnetic susceptibility. Figure 2a depicts the temperature dependence of the magnetic susceptibility $(\chi(T))$ of BMTO in a magnetic field $\mu_{0} H=1$ T. In order to estimate the effective magnetic moment $\mu_{e f f}$ and the Curie-Weiss temperature $\left(\theta_{C W}\right)$, the inverse magnetic susceptibility, was fitted (see right y-axis of Fig. $2 \mathrm{a}$ ) with the Curie-Weiss model $\chi=\chi_{0}+\frac{C}{T-\theta_{C W}}$, where $\chi_{0}$ is the temperature independent contribution due to core diamagnetism and van Vleck paramagnetism, $C$ is the Curie-constant and $\theta_{C W}$ is the Curie-Weiss temperature. The Curie-Weiss fitting in the high-temperature range $150 \mathrm{~K} \leq T \leq 340 \mathrm{~K}$ yields $C=4.45 \mathrm{~cm}^{3} \mathrm{~K} / \mathrm{mol}, \chi_{0}=4.5 \times 10^{-5}$ $\mathrm{cm}^{3} / \mathrm{mol}$ and $\theta_{C W}=-152 \mathrm{~K}$. The relatively large and negative value of $\theta_{C W}$ reveals the presence of strong antiferromagnetic exchange interaction between $\mathrm{Mn}^{2+}$ spins. The calculated effective moment $\mu_{e f f}=\sqrt{8 C}=5.97 \mu_{B}$ per $\mathrm{Mn}$ atom is very close to the expected moment $\mu_{\text {eff }}=g \sqrt{S(S+1)} \mu_{B}=5.92 \mu_{B}$ for the high-spin state $(S$ $=5 / 2)$ of $\mathrm{Mn}^{2+}$ assuming the $g$ factor $g=2^{46}$. The high spin state is further confirmed by the DFT calculations. The corresponding effective moment gives the Landé $\mathrm{g}$ factor $g=2.018$, a similar $g$ value was also determined in the triangular lattice $\mathrm{Ba}_{3} \mathrm{MnSb}_{2} \mathrm{O}_{9}$ by ESR ${ }^{47}$. With decreasing temperature the $\chi(T)$ data start deviating from the Curie-Weiss law and show an anomaly at $20 \mathrm{~K}$ which suggests that antiferromagnetic long-range order sets in at this temperature. Similar behavior was also seen in several other frustrated triangular lattice systems ${ }^{48}$. Indeed, other compounds in this series of double perovskites also show long-range antiferromagnetic ordering around $20 \mathrm{~K}$, e.g., $\mathrm{Sr}_{2} \mathrm{CuTeO}_{6}$ and $\mathrm{Pb}_{2} \mathrm{MnTeO}_{6}{ }^{35}$, 49 . The strength of frustration in the present antiferromagnet is quantified by the frustration parameter $f=\left|\theta_{C W}\right| / T_{N} \approx 7$, which suggests the existence of moderate frustration in the host magnetic lattice. As shown in Fig. $2 \mathrm{~b}, \chi(T)$ data for all fields up to $7 \mathrm{~T}$ are very similar in magnitude and we observed no shift in anomaly with the applied field up to $7 \mathrm{~T}$. Absence of any hysteresis in magnetization curve at $5 \mathrm{~K}$ (Fig. 2c) excludes any ferromagnetic component, either being intrinsic or due to a tiny amount of impurity 

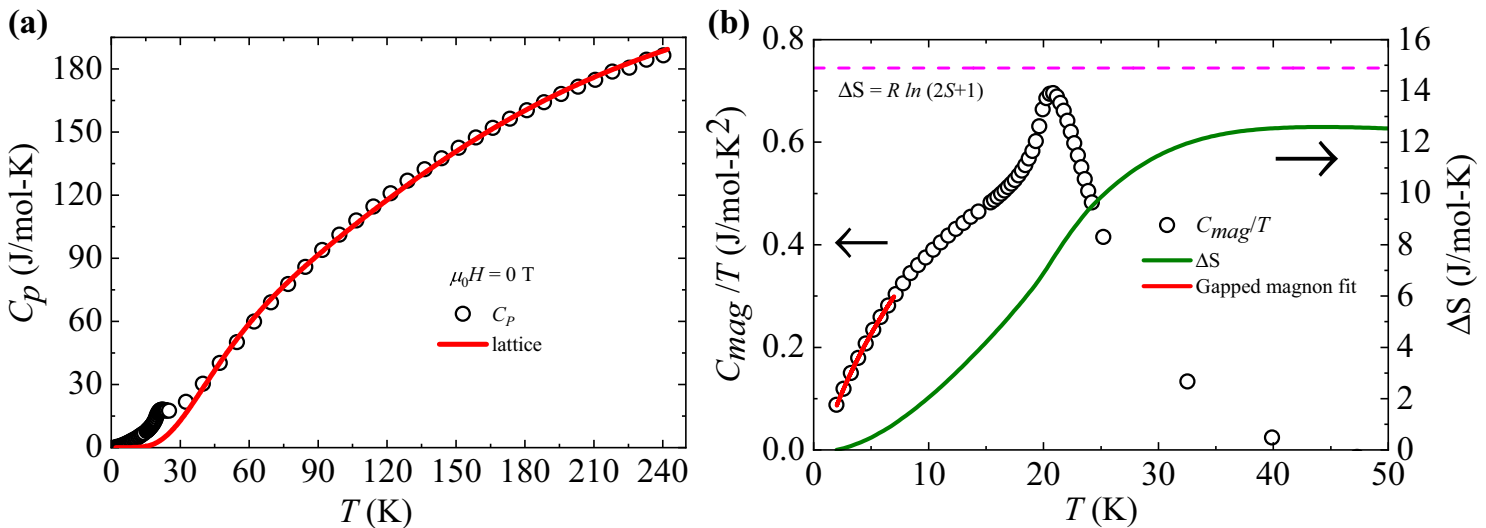

Figure 3. (a) The temperature dependence of specific heat $C_{p}(T)$ of polycrystalline samples of BMTO in the temperature range $2 \mathrm{~K} \leq T \leq 250 \mathrm{~K}$ at zero applied field. The solid line is the fit of $C_{p}(T)$ data to Debye + Einstein model accounting for the lattice contribution to specific heat. (b) The temperature dependence of magnetic specific heat $\left(C_{m a g}(T) / T\right)$ of BMTO in the temperature range $2 \mathrm{~K} \leq T \leq 50 \mathrm{~K}$ (left y-axis) and the calculated entropy change with temperature (right y-axis).

phase of $\mathrm{Mn}_{3} \mathrm{O}_{4}{ }^{50}$. The reduced magnetic moment compared to saturation moment $5.92 \mu_{B}\left(\mathrm{Mn}^{2+}, S=5 / 2\right)$ at 7 $\mathrm{T}$ is consistent with the presence of strong antiferromagnetic exchange interactions between $\mathrm{Mn}^{2+}$ spins.

Specific heat. In order to provide further evidence of long-range magnetic order, we have measured the temperature dependence of specific heat $\left(C_{p}(T)\right)$ of BMTO in zero field in the temperature range $2 \mathrm{~K} \leq T \leq$ $250 \mathrm{~K}$. A lambda-like anomaly appears at $T_{N}=20 \mathrm{~K}$, which is the same temperature at which we observed an anomaly in $\chi(T)$. This confirms the occurrence of an antiferromagnetic long-range order in BMTO at this temperature. The absence of any anomaly at $42 \mathrm{~K}$, which is the transition temperature of $\mathrm{Mn}_{3} \mathrm{O}_{4}$, indicates $\mathrm{BMTO}$ is free from minor impurity phase of $\mathrm{Mn}_{3} \mathrm{O}_{4}{ }^{51}$. An estimate of the associated magnetic contribution to the specific heat data of BMTO is obtained after subtraction of lattice contribution from the total specific heat data, i.e., $C_{\text {mag }}(T)=C_{p}(T)-C_{\text {latt }}(T)$, where $C_{\text {mag }}(T)$ and $C_{\text {latt }}(T)$ are the magnetic and lattice specific heat, respectively. In the absence of a suitable non-magnetic analog of BMTO, we model the lattice contribution as ${ }^{52}$

$$
\begin{gathered}
C_{\text {latt }}(T)=C_{D}\left[9 k_{B}\left(\frac{T}{\theta_{D}}\right)^{3} \int_{0}^{\theta_{D} / T} \frac{x^{4} e^{x}}{\left(e^{x}-1\right)^{2}} d x\right] \\
+\sum_{i=1}^{3} C_{E_{i}}\left[3 R\left(\frac{\theta_{i}}{T}\right)^{2} \frac{\exp \left(\frac{\theta_{E_{i}}}{T}\right)}{\left(\exp \left(\frac{\theta_{E_{i}}}{T}\right)-1\right)^{2}}\right]
\end{gathered}
$$

which includes a Debye term and three Einstein terms. In Eq. (1) $\theta_{D}$ is the Debye temperature, $\theta_{i}$ are the Einstein temperatures of the three modes, $R$ and $k_{B}$ are the molar gas constant and Boltzmann constant, respectively. As depicted in Fig. 3a, the experimental data show good agreement with the model for temperatures above $40 \mathrm{~K}$ for $\theta_{D}=324 \mathrm{~K}, \theta_{E_{1}}=128 \mathrm{~K}, \theta_{E_{2}}=194 \mathrm{~K}$, and $\theta_{E_{3}}=645 \mathrm{~K}$. In the fit the coefficients were fixed in the ratio $C_{D}: C_{E_{1}}: C_{E_{2}}$ $: \mathrm{C}_{E_{3}}=1: 1: 3: 5$ as in BMTO the number of acoustic and optical modes of lattice vibration has the ratio of $1: 9^{53}$. The one Debye term corresponds to the acoustic mode and three Einstein terms approximate all optical modes. After subtracting the lattice contribution, the magnetic contribution to specific heat $C_{m a g}(T)$ is obtained and shown in Fig. 3b. There is a clear anomaly in $C_{m a g}(T) / T$ at $T_{N}=20 \mathrm{~K}$, which suggests that $\mathrm{Mn}$-Mn exchange interaction connectivity in BMTO is essentially $3 D$. Next, we have calculated the entropy change $(\Delta S(T))$ by integrating $C_{\text {mag }}(T) / T$ over the temperature range from 2 to $50 \mathrm{~K}$ as shown in Fig. $3 \mathrm{~b}$. It is noticed that the rise of entropy change with increasing temperature saturated to a value of $12.34 \mathrm{~J} / \mathrm{mol}-\mathrm{K}$ at $50 \mathrm{~K}$, which is somewhat lower than the expected value of the total entropy $14.9 \mathrm{~J} / \mathrm{mol} . \mathrm{K}(R \ln (2 S+1))$ for the high-spin $S=5 / 2$ state of $\mathrm{Mn}^{2+}$ ions. Thus, we recovered $82 \%$ of the total entropy and the missing $18 \%$ is most likely due to over-estimation of the lattice contribution to total specific heat and thus underestimation of short-range spin correlations above $T_{N}$. Below the transition temperature, the lattice contribution to the specific heat becomes practically negligible so the measured specific heat is of magnetic origin. At low temperatures up to $T_{N}$, approximately $50 \%$ of the entropy is recovered, suggesting that the other $50 \%$ is due to short-range spin correlations that develop already above $T_{N}$. In order to investigate the nature of magnetic excitations in the ground state, the low temperature (up to $\sim$ $T_{N} / 3$ ) magnetic specific heat data are fitted with a phenomenological model ${ }^{5-56}$

$$
C_{\text {mag }}(T)=\alpha T^{n} \exp (-\Delta / T)
$$

where $\alpha$ and $n$ are constants and $\Delta$ is the gap between lower band and upper band of closely spaced energy levels. A similar empirical formula was employed to describe the gapped magnon excitations of $\alpha-\mathrm{RuCl}_{3}$ in the ground state $^{56,57}$. The fit yields a gap $\Delta / k_{B} \approx 1.4 \pm 0.1 \mathrm{~K}$ in the magnetic excitation spectrum. The presence of small gap is attributed to an easy-axis anisotropy term in the spin Hamiltonian ${ }^{55,58}$. 

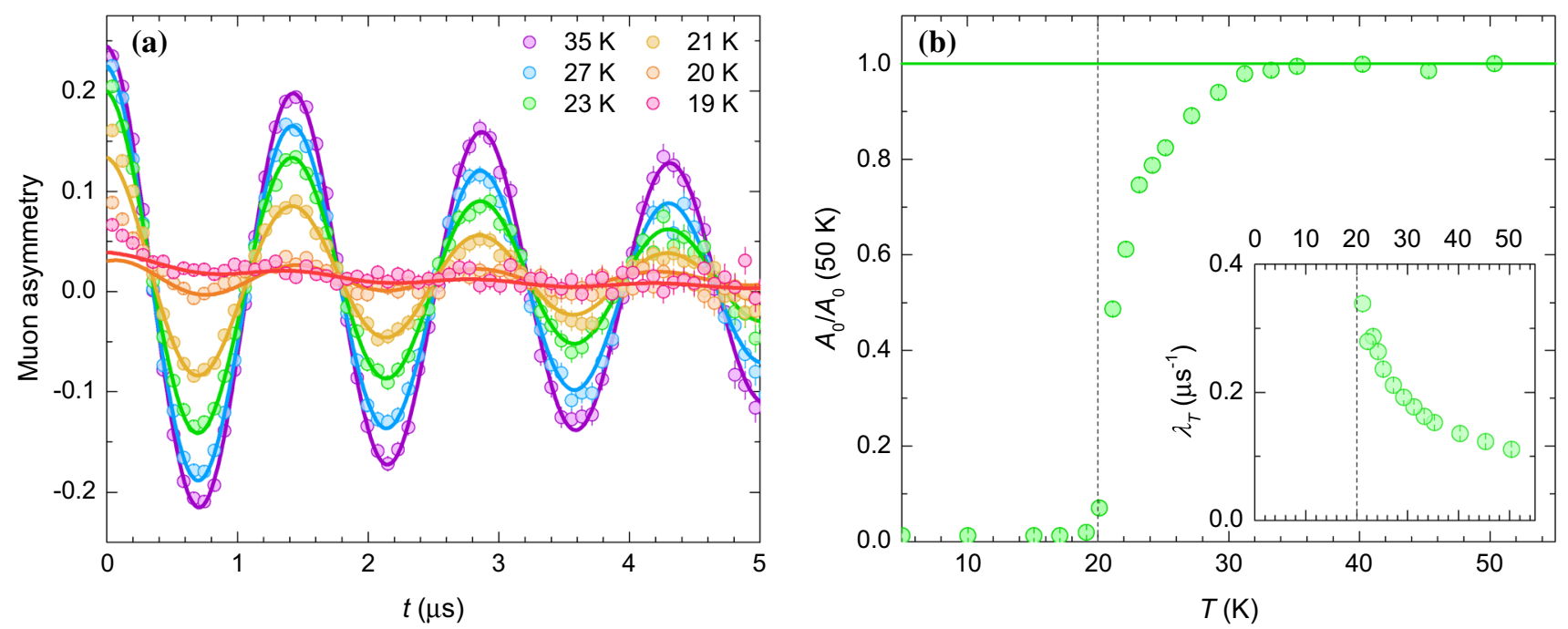

Figure 4. (a) The time dependence of the muon asymmetry in BMTO in a transverse field $B_{\mathrm{TF}}=5 \mathrm{mT}$ at various temperatures (symbols). The solid lines are fits with the model (3) for $t>0.3 \mu \mathrm{s}$. (b) The relative amplitude of the signal oscillating with the frequency $\gamma_{\mu} B_{\mathrm{TF}} /(2 \pi)$ corresponding to the fraction of muons not detecting static internal fields of electronic origin in BMTO. The inset shows the temperature dependence of the transverse muon relaxation rate of the oscillating signal. The vertical dashed line shows the position of $T_{N}$.

Muon spin relaxation ( $\mu$ SR). Transverse field (TF) $\mu$ SR measurements are a very efficient probe of magnetic ordering and spin correlations. In the absence of static internal magnetic fields of electronic origin, the muon asymmetry precesses in a weak external transverse field $B_{\mathrm{TF}}$ with the frequency $\gamma_{\mu} B_{\mathrm{TF}} /(2 \pi)$, where $\gamma_{\mu}=2 \pi \times 135.5 \mathrm{MHz} / \mathrm{T}$ is the muon gyromagnetic ratio. Muons experiencing additional static internal fields, which are in insulators usually in the range between a few tens and a few hundreds of $\mathrm{mT}^{59}$, oscillate much faster and lead to a strongly damped signal that is observable only at very short times. Except from these short times, muon asymmetry follows the general form

$$
A_{T F}(t)=A_{0} \cos \left(\gamma_{\mu} B_{\mathrm{TF}} t\right) \mathrm{e}^{-\lambda_{T} t}+A_{1},
$$

where the amplitude $A_{0}$ describes the volume fraction of the sample that experiences zero static internal fields and $A_{1}>0$ arises from the ordered part of the sample with a component of a local field parallel to the initial muon polarization. The temperature dependence of the TF $\mu$ SR asymmetry in BMTO with corresponding fits of the model (3) is shown in Fig. 4a. The relative amplitude of the signal oscillating with the frequency $\gamma_{\mu} B_{\mathrm{TF}} /(2 \pi)$, i.e., the volume fraction of the muons not experiencing sizeable static internal magnetic fields, starts decreasing from unity already below $35 \mathrm{~K} \simeq 2 T_{N}$ and quickly drops towards zero when the temperature approaches $T_{N}$ (Fig. $4 \mathrm{~b}) . A_{0}(T) / A_{0}(50 \mathrm{~K})<1$ indicates the presence of static internal fields, which we attribute to short-range ordering for $T>T_{N}$ and long-range ordering for $T<T_{N}$. We note that in BMTO diffuse neutron scattering originating from the same $Q$ positions as spin waves below $T_{N}$ is found at temperatures far above $T_{N}{ }^{37}$, therefore our confirmation of the short-range order nicely complements these results. The transverse muon spin relaxation rate $\lambda_{T}$ (inset in Fig. 4b), which measures the width of the distribution of static fields for the component with no net internal field, increases when temperature approaches $T_{N}$.

Next, we determine the static internal magnetic fields $B_{\mu}$ in BMTO below $T_{N}$ more precisely from zero field (ZF) $\mu \mathrm{SR}$ measurements, where the frequency of oscillations in muon asymmetry is directly given by these fields, $v_{\mu}=\gamma_{\mu} B_{\mu} /(2 \pi)$. Indeed, high-frequency oscillations develop in the muon asymmetry below $T_{N}$ due to magnetic ordering (Fig. 5a). The corresponding experimental curves can be fit at short times with a model including two distinct muon stopping sites,

$$
\begin{aligned}
A_{Z F}^{s}(t)= & A_{0} f\left[\frac{2}{3} \cos \left(\gamma_{\mu} B_{\mu 1} t\right) \mathrm{e}^{-\lambda_{T 1} t}+\frac{1}{3}\right] \\
& +A_{0}(1-f)\left[\frac{2}{3} \cos \left(\gamma_{\mu} B_{\mu 2} t\right) \mathrm{e}^{-\lambda_{T 2} t}+\frac{1}{3}\right] .
\end{aligned}
$$

Here, the constant " $1 / 3$-tail" for each site corresponds to the projection of the initial polarization in a powder sample on the internal magnetic field, which does not precess, while the oscillating part is due to the perpendicular component ${ }^{59}$. The internal fields at the two muons stopping sites at $1.6 \mathrm{~K}$ amount to $B_{\mu 1}=0.63 \mathrm{~T}$ and $B_{\mu 2}=0.38 \mathrm{~T}$, while large relaxation rates $\lambda_{T 1} \simeq \lambda_{T 2} \sim 60(10) \mu \mathrm{s}^{-1}$ indicate relatively broad distributions of internal fields. The temperature dependence of the average internal fields (Fig. 5b) corresponds to the evolution of the order parameter and a critical behavior is observed close to $T_{N}$. We find that down to $14 \mathrm{~K}$ the internal fields at both muons stopping sites scale like $B_{\mu}=B_{\mu}^{0}\left(1-T / T_{N}\right)^{\beta}$, with the critical exponent $\beta=0.13(1)$ (inset in Fig. 5b). On the low temperature side, on the other hand, the fields at both sites remain saturated up 

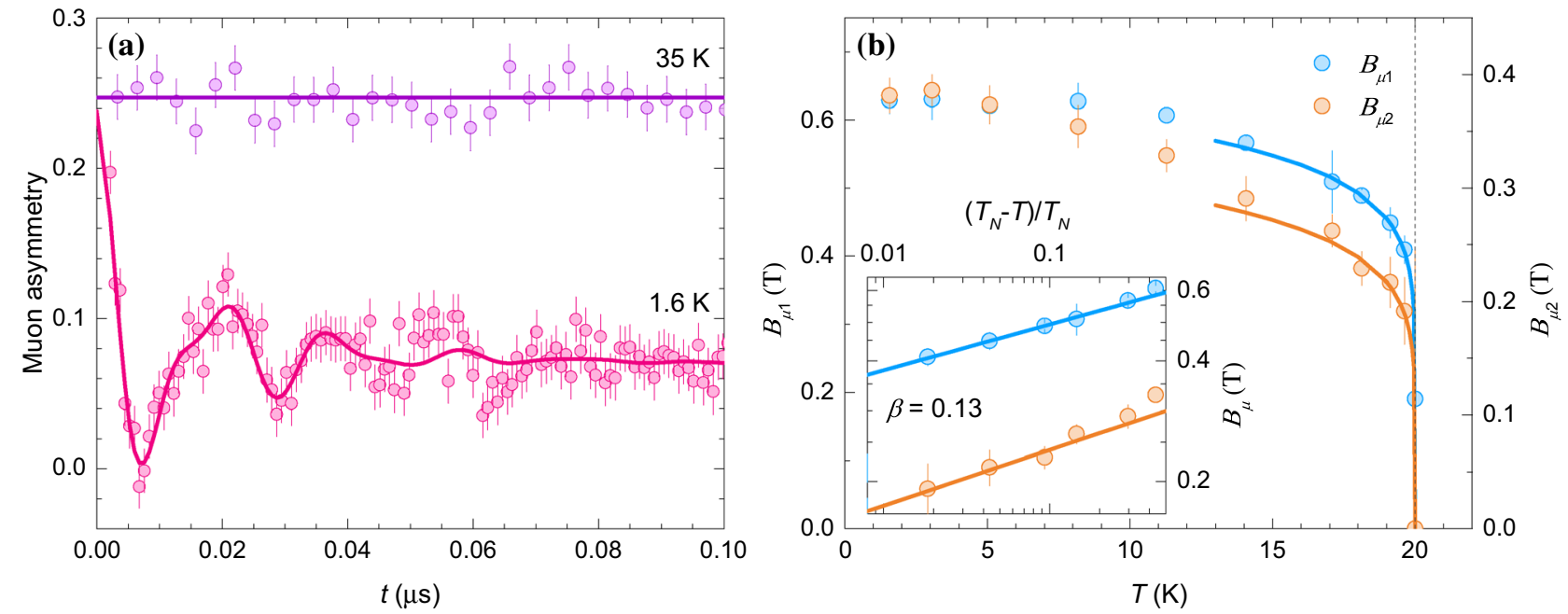

Figure 5. (a) Zero-field muon asymmetry in BMTO at short times above and below $T_{N}=20 \mathrm{~K}$ (symbols). The solid lines are fits with the model (4). (b) The temperature dependence of the internal fields at the two muon stopping sites. The solid lines demonstrate critical scaling of internal fields with critical exponent $\beta=0.13$ (see text for details), which is further highlighted on a log-log scale in the inset. The vertical dashed line shows the position of $T_{N}$.
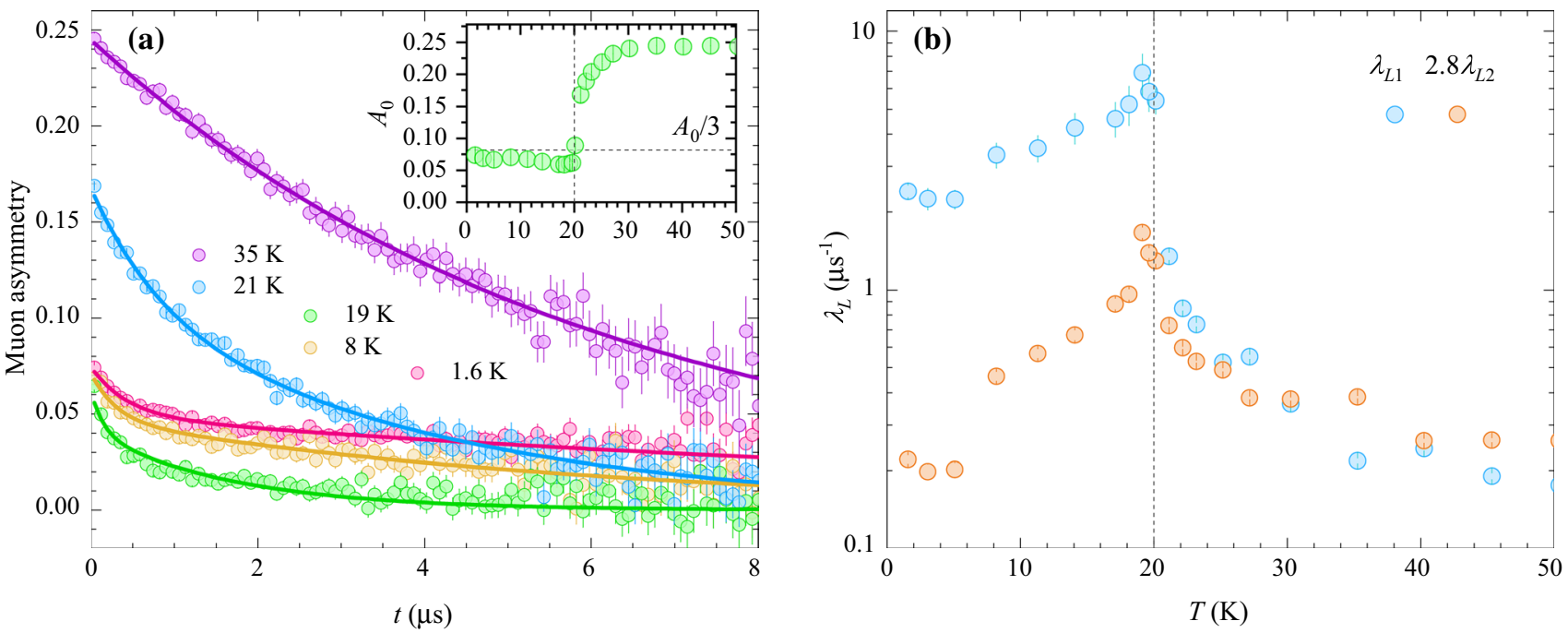

Figure 6. (a) Zero-field muon asymmetry in BMTO at various temperatures (symbols). The inset shown the initial asymmetry of the non-oscillating signal. The solid lines are fits with the model (5). (b) The temperature dependence of the longitudinal muon spin relaxation rates at the two muon stopping sites in BMTO. The vertical dashed line shows the position of $T_{N}$.

to $5 \mathrm{~K}$, while at higher temperatures their magnitude decreases due to magnon excitations. The decrease of the field at site 2 seems to be more enhanced that at site 1 , which is probably due to different filtering of magnons at both stoping sites that are unknown for BMTO. At $T_{N}$ the static internal fields vanish, contrary to the refined magnetic moment deduced from neutron diffraction, which exhibits a smooth evolution across the transition temperature ${ }^{37}$. The fraction of the muon stopping site with the higher internal field value is $f=0.32(5)$ and is temperature independent. On a timescale longer than $0.1 \mu \mathrm{s}$, the fast oscillations due to static internal magnetic fields below $T_{N}$ are averaged out, so that only the "1/3-tail" is seen in ZF muon asymmetry (Fig. 6a). This tail exhibits pronounced relaxation even at the lowest temperature of $1.6 \mathrm{~K}$, i.e., well below $T_{N}$, which is due to the dynamics of the local fields. In fact, the ZF muon asymmetry on the long time scale can be fit with the same model

$$
A_{Z F}^{l}(t)=A_{0} f \mathrm{e}^{-\lambda_{L 1} t}+A_{0}(1-f) \mathrm{e}^{-\lambda_{L 2} t},
$$

at all temperatures, where the two terms again correspond to the two muon stopping sites and the parameter $f=0.32(5)$ is fixed to the value determined above from the oscillating component on the shorter time scale. The initial asymmetry falls from the high-temperature value of $A_{0}=0.245$ to about $A_{0} / 3$ (inset in Fig. 6a), as expected. The decrease of this parameter is due to ordering effects. The begining of the decrease is again observed 


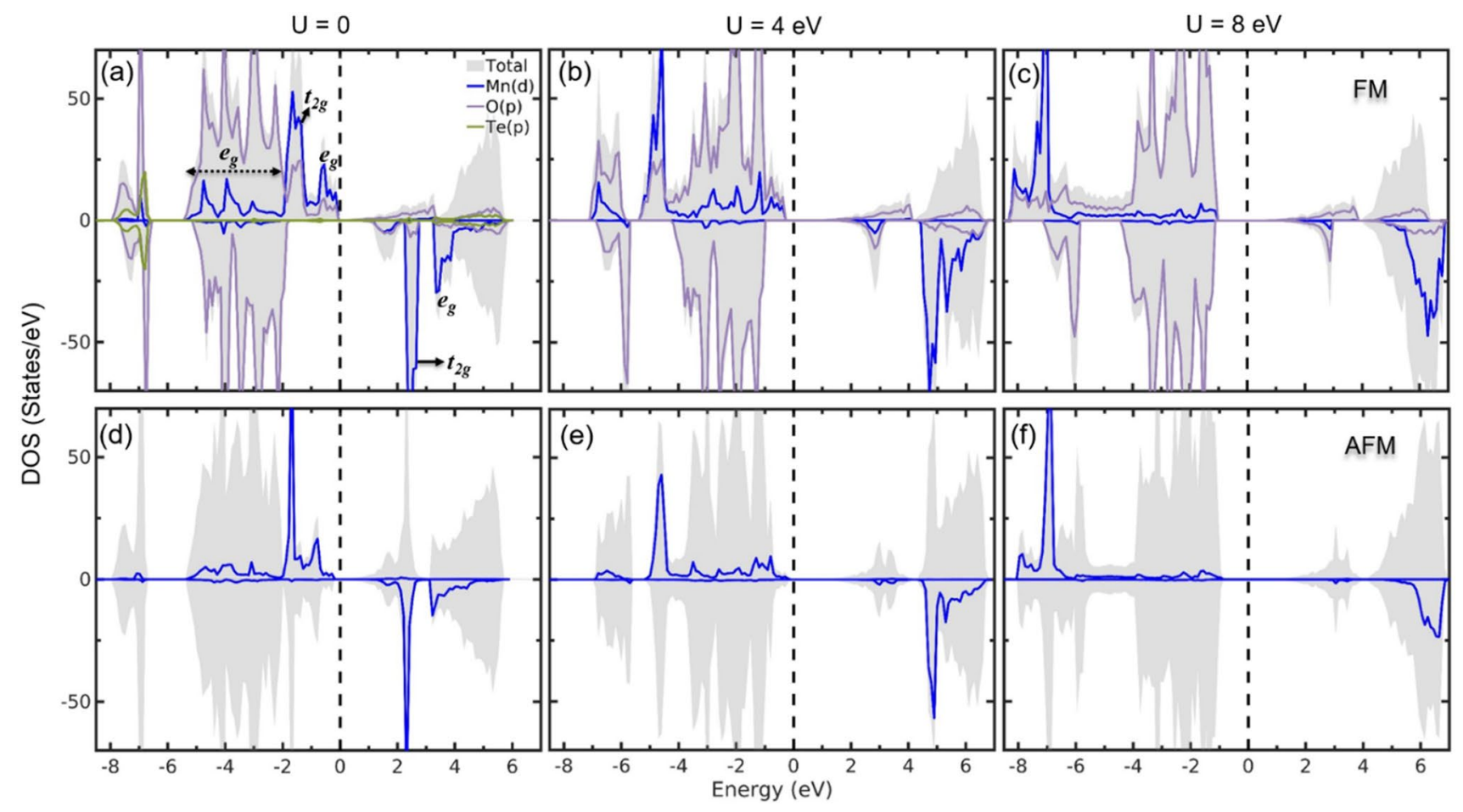

Figure 7. (a) The total and partial densities of states (DOS) for the ferromagnetic (FM) and the energetically most stable antiferromagnetic (AFM) configuration (AFM3, see Fig. 8e) as a function of the Hubbard $U$. For clarity, in the AFM configuration, the Mn- $d$ states of only one spin-sublattice is shown. For the opposite spinsublattice, the corresponding DOS is simply a mirror image.

already below $35 \mathrm{~K}$ and becomes very pronounced in close vicinity of $T_{N}$, mimicking the change of the amplitude of the TF signal shown in Fig. $4 \mathrm{~b}$. The longitudinal muon relaxation rates $\lambda_{L 1}$ and $\lambda_{L 2}$ exhibit divergent behavior at $T_{N}$ (Fig. 6b), which is a typical fingerprint of critical slowing down of spin fluctuations. Above $T_{N}$ the ratio of the relaxation rates of the two components scales with the ratio of the squares of the internal fields in the longrange ordered phase, $\lambda_{L 1} / \lambda_{L 2}=\left(B_{\mu 1} / B_{\mu 2}\right)^{2}=2.8$ (Fig. $6 \mathrm{~b}$ ). As the muon spin relaxation rate is proportional to the square of the fluctuating fields in the fast-fluctuation regime corresponding to the paramagnetic phase ${ }^{59}$, this experimental scaling firmly validates our analysis with two muon stopping sites in BMTO and the determined fraction $f=0.32(5)$ of muons stopping at the site with larger internal fields. Below $T_{N}$ the longitudinal muon spin relaxation is due to collective excitations and the ratio $\lambda_{L 1} / \lambda_{L 2}$ increases by an order of magnitude. Importantly, we find that the dynamics of local fields persists down to the lowest temperatures, as observed in various different frustrated spin systems ${ }^{60-62}$.

Electronic structure calculations. The crystal structure of BMTO can be described as alternate stacking of layers of $\mathrm{TeO}_{6}$ and $\mathrm{MnO}_{6}$ octahedra, and the neighboring layers are connected through corner sharing oxygen as shown in Fig. 1a. However, the electronic structure, presented in Fig. 7 through total and partial densities of states (DOS), shows that the Te- $p$ state is almost completely occupied and lies around $7 \mathrm{eV}$ below the Fermi energy $\left(E_{F}\right)$. Therefore, as Te is inactive, from the electronic and magnetic structure point of view this compound can be treated as an open spaced structure in the sense that the minimum Mn-Mn separation, both inter-layer and intra-layer, is $\sim 5.8 \AA$ which is roughly double than that of the closed packed transition metal perovskites. Here, the electronic structure of the system is supposed to be nearly a sum of the electronic structure of the individual $\mathrm{MnO}_{6}$ octahedra ${ }^{63}$. To verify this we first examine the DOS within the independent electron approximation $(U=0)$ which are shown in Fig. 7a. Here, we observe that due to crystal-field splitting, the $\mathrm{Mn}-d$ states split into triply degenerate $t_{2 g}$ and doubly degenerate $e_{g}$ states. Due to stronger axial interactions, the $e_{g}$ states have reasonable overlapping with the $\mathrm{O}-p$ states, which dominate the energy window -5 to $-2 \mathrm{eV}$ with respect to $E_{F}$. Due to $2+$ charge state for $\mathrm{Mn}$, the $d$-orbitals are half-occupied and stabilize in a high-spin states $(S=5 / 2)$ where the $d$-orbitals in the spin minority channel are completely unoccupied. The $S=5 / 2$ state is also confirmed from the magnetization measurement discussed earlier. For such a spin-state, the spin splitting is strong enough to create a band gap even for $U=0^{63}$. With inclusion of strong correlation effect (finite $U$ ), the $\mathrm{Mn}-d$ states are pushed to lower energies in the valence band and to higher energies in the conduction band to widen the bandgap (see Fig. $7 \mathrm{a}-\mathrm{c}$ ). The $\mathrm{O}-p$ states now dominate the valence band near $E_{F}$ which implies that BMTO is a charge transfer insulator ${ }^{64}$ which favors antiferromagnetic (AFM) ordering. The total and Mn-d DOS for the stable AFM ordering (see Fig. 7e) are shown in the lower panel of Fig. 7d-f. As both FM and AFM ordering makes the system insulating, we infer that this is primarily a weakly coupled classical spin system. The strength of the coupling is discussed next. 
(a)

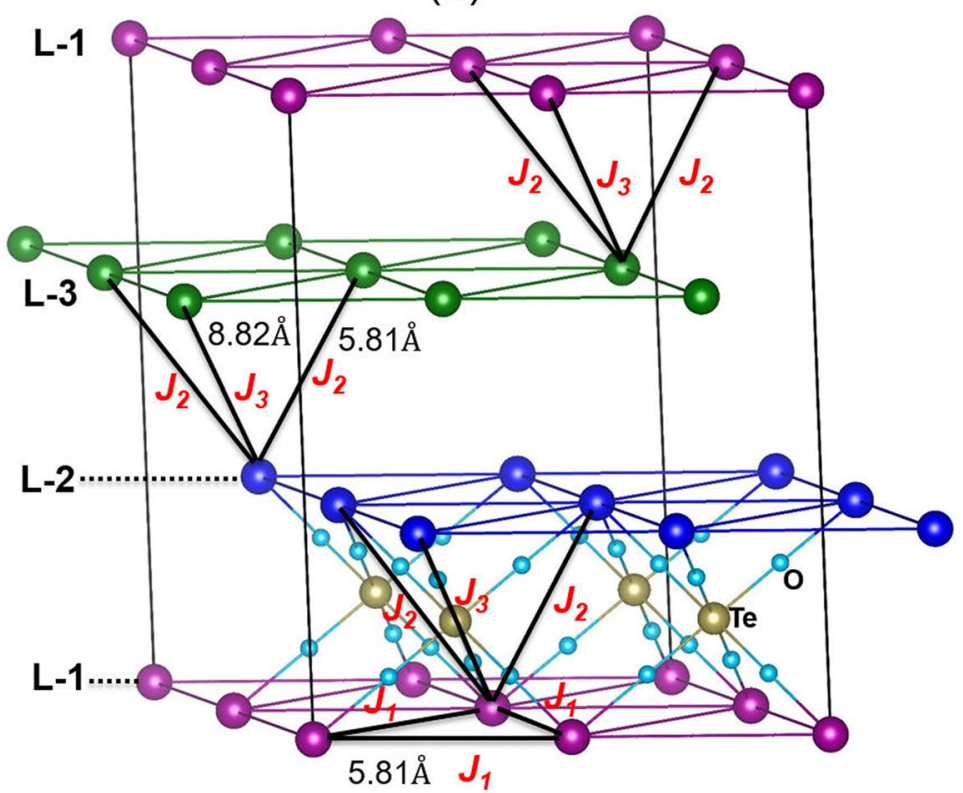

(b) FM

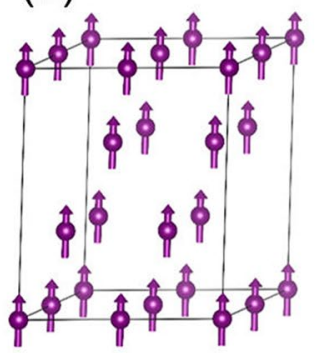

(d) AFM2

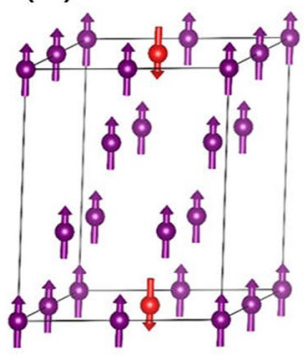

(c) AFM1

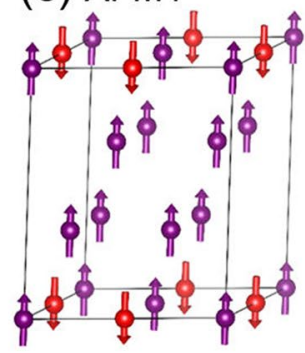

(e) AFM3

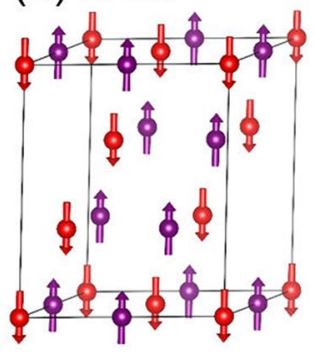

Figure 8. (a) The dominant exchange interaction paths $J_{1}, J_{2}$ and $J_{3}$ for the $\mathrm{Mn}$ spins in the layered compound BMTO. $J_{1}$ represents the in-plane nearest neighbor $\left(5.81 \AA\right.$ ) exchange interaction, while $J_{2}$ and $J_{3}$ correspond to out-of-plane nearest and next-nearest interactions with Mn-Mn separations at $5.81 \AA$ and $8.22 \AA$, respectively. The $J_{1}$ forms a triangular lattice in the plane. To estimate the strengths of the $J_{i}$ 's, several total energy calculations were carried out on four spin arrangements, defined as FM, AFM1, AFM2, and AFM3 (b-e).

Magnetic interactions in BMTO. The experimental results presented in this work imply dominant antiferromagnetic interactions through $\theta_{C W}$. There are three dominant exchange interaction paths $\left(J_{1}, J_{2}, J_{3}\right)$ in this compound which demonstrates a hexagonal $\mathrm{ABC}$ stacking pattern as shown in Fig. 8a. The $J_{1}$ represents the intra-plane nearest-neighbor interaction for which the $\mathrm{Mn}-\mathrm{Mn}$ distance is $5.81 \AA$, whereas $J_{2}$ represents the inter-plane nearest-neighbor interaction $\left(d_{M n-M n}=5.81 \AA\right)$. The 3rd nearest-neighbor interaction $(8.22 \AA)$ is considered by the $J_{3}$ term. Here, we shall examine the strengths of these $J_{i}$ 's through a spin-dimer analysis using Noodlemann's broken-symmetry method ${ }^{65,66}$. According to this method, the energy difference between the high spin (HS) and low spin (LS) states for a spin dimer is given by

$$
E_{H S}-E_{L S}=\frac{1}{2}\left(S_{\max }\right)^{2} J
$$

where $J$ is related to the spin-dimer Hamiltonian, $\hat{H}=J \hat{S}_{1} \cdot \hat{S}_{2}$, with $S_{\text {max }}$ being the maximum spin of the dimer. As we have a Mn-Mn spin dimer, both sites of the dimer have five unpaired electrons. Therefore, Eq. (6) reduces to

$$
E_{H S}-E_{L S}=\frac{25}{2} J
$$

where, $E_{H S}$ and $E_{L S}$ can be estimated from the DFT calculations as discussed below.

To evaluate exchange constants in the framework of DFT, one needs to design several possible magnetic configurations, and calculate the total energies. The relative energy differences among them are expressed in terms of $J_{i}$ 's leading to a set of linear equations. The magnetic configurations (FM, AFM1, AFM2, and AFM3), considered here are designed on a $2 \times 2 \times 2$ supercell as shown in Fig. 8b-e. The total energy of each configuration is estimated with the sum of all exchange paths which yield the following set of equations:

$$
\begin{gathered}
E_{F M}=(25 / 4)\left(36 J_{1}+36 J_{2}+36 J_{3}\right), \\
E_{A F M 1}=(25 / 4)\left(20 J_{1}+12 J_{2}+12 J_{3}\right), \\
E_{A F M 2}=(25 / 4)\left(24 J_{1}+24 J_{2}+24 J_{3}\right), \\
E_{A F M 3}=(25 / 4)\left(-12 J_{1}-12 J_{2}+36 J_{3}\right),
\end{gathered}
$$

Hence, by solving the above equations, we have estimated the $J_{i}$ 's as a function of $U$ and plotted them in Fig. 9. While the dominant interactions $J_{1}$ and $J_{2}$ are antiferromagnetic, $J_{3}$ corresponds to a weakly ferromagnetic coupling. This is due to the fact that $J_{3}$ is formed by a linear $\mathrm{Mn}-\mathrm{O}-\mathrm{Te}-\mathrm{O}-\mathrm{Mn}$ path, where the axial $\mathrm{e}_{g}$ $-\mathrm{O}-\mathrm{p}-\mathrm{Te}-\mathrm{p}-\mathrm{O}-\mathrm{p}-\mathrm{e}_{\mathrm{g}}$ covalent interaction is formed leading to a double-exchange ferromagnetic interaction. 


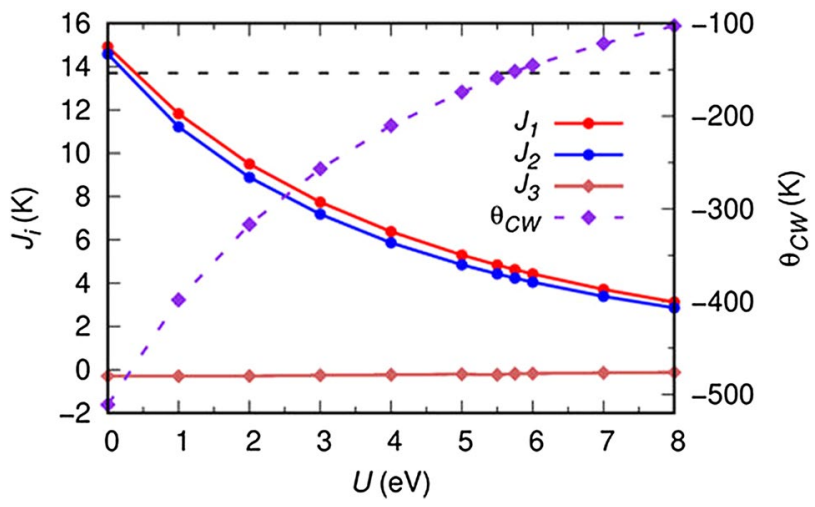

Figure 9. The magnetic exchange-coupling constants of $\mathrm{Mn}$ atoms for intra-layer $J_{1}$, and inter-layer $J_{2}$ and $J_{3}$ as well as $\theta_{C W}$ as a function of Hubbard $U$. The experimental $\theta_{C W}$ value $(-152 \mathrm{~K})$ is shown as dashed line, which matches the calculation for $U=5.75 \mathrm{eV}$.

However, since Te-p states form a nearly closed shell configuration, the interaction is very weak. Although the exact values of the exchange constants $J_{i}$ depend on the Hubbard repulsion parameter $U$, we can evaluate their strength by comparison with the experimentally obtained Curie-Weiss temperature, as recently demonstrated for another frustrated lattice ${ }^{67}$. The Curie-Weiss temperature is given by $\theta_{C W}=-\frac{S(S+1)}{3 k_{B}} \sum_{i} Z_{i} J_{i}$, where $Z_{i}$ represents the coordination numbers of $\mathrm{Mn}$ atom for each interaction. By plotting $\theta_{C W}$ as a function of $U$ (see Fig. 9) we find a match with the experimental value of $-152 \mathrm{~K}$ for $U=5.75 \mathrm{eV}$ for which $J_{1}=4.6 \mathrm{~K}, J_{2}=4.2 \mathrm{~K}$ and $J_{3}=-0.2$ $\mathrm{K}$, yielding $J_{1}: J_{2}: J_{3}=1: 0.92:-0.04$. These values are slightly larger than those $\left(J_{1}=J_{2}=3.1 \mathrm{~K}\right.$ and $\left.J_{3}=-0.6 \mathrm{~K}\right)$ found from the fit of spin waves detected by inelastic neutron scattering ${ }^{38}$. The latter values however underestimate the Curie-Weiss temperature.

\section{Discussion}

A conventional $3 D$ antiferromagnet exhibits a symmetry breaking phase transition at a temperature close to the Curie-Weiss temperature, but the here investigated compound BMTO shows long-range order only at $T_{N}=20$ $\mathrm{K}$ despite much larger $\theta_{C W} \sim-152 \mathrm{~K}$. This suggests that BMTO is a moderately frustrated antiferromagnet with frustration index $f=7$. In this system, two structural reasons could explain the observed long-range order: (1) Magnetic ions are in the high-spin state $(S=5 / 2)$ and quantum fluctuations are less pronounced even though the $\mathrm{Mn}^{2+}$ ions are arranged in $2 D$ triangular plane; (2) The intra-plane and the inter-plane nearest-neighbor distances are the same which allows for sizeable inter-plane interactions that forces the system to undergo a long-range magnetic ordering. The origin of the magnetic ordering in this strongly correlated system is examined within the framework of DFT. Our calculations of exchange interaction reveal dominant antiferromagnetic intra-layer exchange coupling $J_{1}=4.6 \mathrm{~K}$ and a comparable inter-layer $J_{2}=0.92 J_{1}$. Furthermore, a very weak ferromagnetic inter-layer interaction exists with third nearest neighbor $\left(J_{3}=-0.04 J_{1}\right)$ due to double-exchange interaction via the linear path $\mathrm{Mn}-\mathrm{O}-\mathrm{Te}-\mathrm{O}-\mathrm{Mn}$. As the strong inter-layer exchange coupling $J_{2}$ is frustrated, it should not be essential for stabilization of $3 D$ order, in accordance with previous investigations of a spatially anisotropic triangular lattice ${ }^{68}$. BMTO should thus behave as an effective $2 D$ rather than a $3 D$ system. Indeed, the analysis of the critical exponent $\beta$ corresponding to the temperature dependence of the order parameter gives $\beta=0.13(1)$. This value is much lower that the critical exponents of unfrustrated $3 D$ systems, where they vary from 0.32 for the Ising spins to 0.36 for the Heisenberg spins, or even the critical exponents of a frustrated stacked triangular lattice, where $\beta=0.30$ for Heisenberg spins and 0.25 for XY spins are governed by spin chirality ${ }^{69}$. The experimental value is, however, very close to the critical exponent of a $2 D$ Ising system, where $\beta=1 / 8$. This might be related to easy-axis anisotropy evidenced by our specific heat measurements. The magnetic specific heat data below the AFM transition are well reproduced with Eq. (2) suggesting the presence of magnon excitations. A broad maximum at $10 \mathrm{~K}$ in magnetic specific heat data suggests the presence of gapped magnon excitation in the ground state. Similar types of broad maxima were also observed in $\mathrm{BiMnVO}_{5}$ and $\mathrm{MnWO}_{4}$ which indicates $\mathrm{Mn}^{2+}$ ions are subjected to anisotropic magnetic interactions ${ }^{55,70}$. In BMTO, the estimated magnon excitation gap is $1.4 \mathrm{~K}$, a similar value of magnon excitation gap is also observed in $\mathrm{MnWO}_{4}{ }^{58}$. The missing of entropy which is estimated as $\sim 18 \%$ of the expected entropy for $S=5 / 2$ system is due to the presence of spin frustration and significant short-range spin correlations between $\mathrm{Mn}^{2+}$ spins already above $T_{N}$, which are however underestimated by our crude modeling of the lattice contribution to the specific heat. In fact, the evolution of $\mu \mathrm{SR}$ spectra in weak transverse field show that short-range ordering effects become apparent already at $35 \mathrm{~K} \simeq$ $2 T_{N}$, and gradually increase as the temperature approaches $T_{N}$. The ratio of the $\mu$ SR amplitudes $A_{0}(T) / A_{0}(50$ $\mathrm{K})<1$ in the temperature range $20 \mathrm{~K} \leq T \leq 35 \mathrm{~K}$ suggests the presence of short-range spin correlation above the antiferromagnetic transition temperature. If there are no significant short-range spin correlations above the transition temperature, volume fraction of the sample does not change above $T_{N}$ as observed in weak transverse field $\mu \mathrm{SR}$ spectra of $\mathrm{LiCrO}_{2}{ }^{71}$. The short-range spin correlations reflect the presence of moderate spin frustration in the magnetic lattice of BMTO. The zero-field $\mu$ SR spectra show that below $35 \mathrm{~K}$ the spin lattice relaxation rate 
gradually increases, which is commonly observed in the vicinity of magnetic phase transition temperature. At $T$ $>35 \mathrm{~K}$, the temperature independent initial asymmetry can be associated with the paramagnetic nature of $\mathrm{Mn}^{2+}$ spin. The position of sharp maximum in the muon relaxation rate and the reduction of initial asymmetry $\left(A_{0}\right)$ to $A_{0} / 3$ both occur at $T=20 \mathrm{~K}$, which confirms a phase transition at this temperatures. Finally, static internal fields are directly observed through the oscillations of muon asymmetry below $T_{N}$ and the evolution of the order parameter is reflected in the temperature dependence of these fields.

\section{Conclusion}

The double perovskite BMTO crystallizes in the trigonal crystal symmetry $\mathrm{R} \overline{3} \mathrm{~m}$, wherein $\mathrm{Mn}^{2+}$ ions form two dimensional triangular layers with sizeable inter-layer exchange coupling. Our comprehensive results, well supported by first principle calculations reveal the presence of antiferromagnetic long-range magnetic order below $T_{N}=20 \mathrm{~K}$. Below $T_{N}$, magnetic specific heat data suggest the presence of magnon excitations with a gap of approximately $1.4 \mathrm{~K}$, which indicates the presence of magnetic anisotropy as commonly observed in classical Heisenberg systems. Our zero-field and weak transverse field $\mu$ SR results provide a concrete evidence of static internal fields in the long-range ordered state below $T_{N}$ and short-range spin correlations above $T_{N}$. $\mu$ SR detects critical slowing-down of spin dynamics at the transition temperature and persistent spin dynamics to the lowest temperatures. Further studies on single crystals are required to shed more insight into the low energy excitations of this double perovskite based frustrated magnet.This frustrated triangular lattice antiferromagnet is also potentially interesting to uncover exotic ground state associated with quenched disorder in triangular lattice by substitution of less electronegative cations at the tellurium site.

\section{Methods}

Sample synthesis and characterization. Polycrystalline samples of BMTO were prepared by a conventional solid state method. Prior to use, we preheated $\mathrm{BaCO}_{3}$ (Alfa Aesar, 99.997 \%), $\mathrm{MnO}_{2}$ (Alfa Aesar, 99.996 $\%$ ) and $\mathrm{TeO}_{2}$ (Alfa Aesar, $99.9995 \%$ ) to remove any moisture. The appropriate stoichiometric mixtures were pelletized and sintered at $1200^{\circ} \mathrm{C}$ for 30 hours with several intermittent grindings. The phase purity was confirmed by the Rietveld refinement of XRD taken on a smartLAB Rigaku X-ray diffractometer with $\mathrm{Cu} \mathcal{K} \alpha$ radiation $(\lambda=$ $1.54 \AA$ ). Magnetization measurements were carried out using a Quantum Design SQUID VSM in the temperature range $5 \mathrm{~K} \leq T \leq 340 \mathrm{~K}$ under magnetic fields $0 \mathrm{~T} \leq \mu_{0} \mathrm{H} \leq 7 \mathrm{~T}$. Specific heat measurements were performed on a Quantum Design Physical Properties Measurement System (QD, PPMS) by thermal relaxation method, in the temperature range $2 \mathrm{~K} \leq T \leq 240 \mathrm{~K}$. $\mu \mathrm{SR}$ measurements were performed using the GPS spectrometer at the Paul Scherrer Institute, Villigen, Switzerland, on a 1-g powder sample in the temperature range $1.6 \mathrm{~K} \leq T \leq 50$ $\mathrm{K}$. The sample was put on a "fork" sample holder and the veto mode was employed, which ensured negligible background signal, as evidenced by the vanishing amplitude of the signal recorded in weak transverse field below $T_{N}(\sim 1 \%$ of the full signal; Fig. 4$)$. The transverse muon-polarization was used in zero applied field (ZF) and in a weak transverse field (TF) of $5 \mathrm{mT}$.

Computational methods. To understand the magnetic interactions in BMTO, DFT calculations have been performed using the plane-wave-pseudopotential approach as implemented in Quantum ESPRESSO ${ }^{72}$. The experimentally obtained structure has been considered for the calculations. The ultra-soft pseudopotentials are used to describe the electron-ion interactions ${ }^{73}$, in which the valence states of $\mathrm{Mn}$ include 15 electrons from $3 s, 3 p, 4 s$ and $3 d$; Ba includes 10 electrons in $5 s, 5 p$, and $6 s$; Te includes 10 electrons in $5 s$ and $5 p$ orbitals; and $\mathrm{O}$ includes 6 electrons from $2 s$ and $2 p$ shells. The exchange-correlation functional is approximated through PBE-GGA functional ${ }^{74}$. The convergence criterion for self-consistent energy is taken to be $10^{-6} \mathrm{Ry}$. A k-mesh of $4 \times 4 \times 2$ is used for the Brillouin zone integration of the supercell of size $2 \times 2 \times 2$. The kinetic energy cut-off for the electron wave functions is set at $30 \mathrm{Ry}$ and the augmented charge density cut-off is set to be $300 \mathrm{Ry}$. We have also performed test calculations with a higher energy cut-off of $40 \mathrm{Ry}$ and charge density cutoff at $400 \mathrm{Ry}$ as well as with a higher k-mesh of $8 \times 8 \times 4$. As the results remain the same below the tolerance level, we have used the lower cut-off and lower k-mesh to reduce the computational time. The strong correlation effect is examined through Hubbard $U$ formalism ${ }^{75}$. The magnetic coupling strengths are evaluated as a function of $U$ in this strongly correlated system.

Received: 23 December 2020; Accepted: 15 February 2021

Published online: 26 March 2021

\section{References}

1. Anderson, P. Resonating valence bonds: A new kind of insulator?. Mater. Res. Bull. 8, 153-160. https://doi.org/10.1016/00255408(73)90167-0 (1973)

2. Balents, L. Spin liquids in frustrated magnets. Nature 464, 199-208. https://doi.org/10.1038/nature08917 (2010).

3. Khuntia, P. et al. Gapless ground state in the archetypal quantum kagome antiferromagnet $\mathrm{ZnCu}_{3}(\mathrm{OH})_{6} \mathrm{Cl}_{2}$. Nat. Phys. 16, 469-474. https://doi.org/10.1038/s41567-020-0792-1 (2020).

4. Khuntia, P. et al. Local magnetism and spin dynamics of the frustrated honeycomb rhodate $\mathrm{Li}_{2} \mathrm{RhO}_{3}$. Phys. Rev. B 96, 094432. https://doi.org/10.1103/PhysRevB.96.094432 (2017).

5. Khuntia, P. Novel magnetism and spin dynamics of strongly correlated electron systems: Microscopic insights. J. Magn. Magn. Mater. 489, 165435. https://doi.org/10.1016/j.jmmm.2019.165435 (2019).

6. Dey, T. et al. Spin-liquid behavior in $J_{\mathrm{eff}}=\frac{1}{2}$ triangular lattice compound $\mathrm{Ba}_{3} \mathrm{IrTi}_{2} \mathrm{O}_{9}$. Phys. Rev. B 86, 140405. https://doi.org/10. 1103/PhysRevB.86.140405 (2012).

7. Khuntia, P., Kumar, R., Mahajan, A. V., Baenitz, M. \& Furukawa, Y. Spin liquid state in the disordered triangular lattice $\mathrm{Sc}_{2} \mathrm{Ga}_{2}$ $\mathrm{CuO}_{7}$ revealed by nmr. Phys. Rev. B 93, 140408. https://doi.org/10.1103/PhysRevB.93.140408 (2016). 
8. Alicea, J., Chubukov, A. V. \& Starykh, O. A. Quantum stabilization of the 1/3-magnetization plateau in $\mathrm{Cs}_{2} \mathrm{CuBa}_{4}$. Phys. Rev. Lett. 102, 137201. https://doi.org/10.1103/PhysRevLett.102.137201 (2009).

9. Zhou, H. D. et al. Successive phase transitions and extended spin-excitation continuum in the $s=\frac{1}{2}$ triangular-lattice antiferromagnet $\mathrm{Ba}_{3} \mathrm{CuSb}_{2} \mathrm{O}_{9}$. Phys. Rev. Lett. 109, 267206. https://doi.org/10.1103/PhysRevLett.109.267206 (2012).

10. Kumar, R. et al. $\mathrm{Sc}_{2} \mathrm{Ga}_{2} \mathrm{CuO}_{7}$ : A possible quantum spin liquid near the percolation threshold. Phys. Rev. B 92, 180411. https://doi. org/10.1103/PhysRevB.92.180411 (2015).

11. Lee, M. et al. Magnetic phase diagram and multiferroicity of $\mathrm{Ba}_{3} \mathrm{MnNb}_{2} \mathrm{O}_{9}$ : A spin- $\frac{5}{2}$ triangular lattice antiferromagnet with weak easy-axis anisotropy. Phys. Rev. B 90, 224402. https://doi.org/10.1103/PhysRevB.90.224402 (2014).

12. Seki, S., Onose, Y. \& Tokura, Y. Spin-driven ferroelectricity in triangular lattice antiferromagnets $\mathrm{ACrO}_{2}(\mathrm{~A}=\mathrm{Cu}, \mathrm{Ag}, \mathrm{Li}$, or $\mathrm{Na})$. Phys. Rev. Lett. 101, 067204. https://doi.org/10.1103/PhysRevLett.101.067204 (2008).

13. Hwang, J. et al. Successive magnetic phase transitions and multiferroicity in the spin-one triangular-lattice antiferromagnet $\mathrm{Ba}_{3}$ $\mathrm{NiNb}_{2} \mathrm{O}_{9}$. Phys. Rev. Lett. 109, 257205. https://doi.org/10.1103/PhysRevLett.109.257205 (2012).

14. Shimizu, Y., Miyagawa, K., Kanoda, K., Maesato, M. \& Saito, G. Spin liquid state in an organic mott insulator with a triangular lattice. Phys. Rev. Lett. 91, 107001. https://doi.org/10.1103/PhysRevLett.91.107001 (2003).

15. Kurosaki, Y., Shimizu, Y., Miyagawa, K., Kanoda, K. \& Saito, G. Mott transition from a spin liquid to a fermi liquid in the spinfrustrated organic conductor $\kappa-(\mathrm{ET})_{2} \mathrm{cu}_{2}(\mathrm{CN})_{3}$. Phys. Rev. Lett. 95, 177001. https://doi.org/10.1103/PhysRevLett.95.177001 (2005).

16. Watanabe, K., Kawamura, H., Nakano, H. \& Sakai, T. Quantum spin-liquid behavior in the spin-1/2 random Heisenberg antiferromagnet on the triangular lattice. J. Phys. Soc. Jpn. 83, 034714. https://doi.org/10.7566/JPSJ.83.034714 (2014).

17. Abdel-Jawad, M., Tajima, N., Kato, R. \& Terasaki, I. Disordered conduction in single-crystalline dimer mott compounds. Phys. Rev. B 88, 075139. https://doi.org/10.1103/PhysRevB.88.075139 (2013).

18. Klanjšek, M. et al. A high-temperature quantum spin liquid with polaron spins. Nat. Phys. 13, 1130-1134. https://doi.org/10.1038/ nphys4212 (2017).

19. Kenzelmann, M. et al. Direct transition from a disordered to a multiferroic phase on a triangular lattice. Phys. Rev. Lett. $98,267205$. https://doi.org/10.1103/PhysRevLett.98.267205 (2007).

20. Ishikawa, H., Okubo, T., Okamoto, Y. \& Hiroi, Z. Kagome-triangular lattice antiferromagnet $\mathrm{NaBa}_{2} \mathrm{Mn}_{3} \mathrm{~F}_{11}$. J. Phys. Soc. Jpn. 83, 043703. https://doi.org/10.7566/JPSJ.83.043703 (2014).

21. Kadowaki, H., Takei, H. \& Motoya, K. Double-q 120 degrees structure in the heisenberg antiferromagnet on rhombohedrally stacked triangular lattice $\mathrm{LiCrO}_{2}$. J. Phys.: Condens. Matter. 7, 6869-6884. https://doi.org/10.1088/0953-8984/7/34/011 (1995).

22. Ishii, R. et al. Successive phase transitions and phase diagrams for the quasi-two-dimensional easy-axis triangular antiferromagnet $\mathrm{Rb}_{4} \mathrm{Mn}\left(\mathrm{MoO}_{4}\right)_{3}$. Europhys. Lett. 94, 17001. https://doi.org/10.1209/0295-5075/94/17001 (2011).

23. Yamaguchi, H., Kimura, S., Ishii, R., Nakatsuji, S. \& Hagiwara, M. Electron spin resonance in the quasi-two-dimensional triangularlattice antiferromagnet $\mathrm{Rb}_{4} \mathrm{Mn}\left(\mathrm{MoO}_{4}\right)_{3}$. J. Phys. Soc. Jpn. 80, 064705. https://doi.org/10.1143/JPSJ.80.064705 (2011).

24. Rawl, R. et al. $\mathrm{Ba}_{8} \mathrm{MnNb}_{6} \mathrm{O}_{24}$ : A model two-dimensional spin- $\frac{5}{2}$ triangular lattice antiferromagnet. Phys. Rev. Mater. 3, 054412. https://doi.org/10.1103/PhysRevMaterials.3.054412 (2019).

25. Chubukov, A. V. \& Jolicoeur, T. Order-from-disorder phenomena in Heisenberg antiferromagnets on a triangular lattice. Phys. Rev. B 46, 11137-11140. https://doi.org/10.1103/PhysRevB.46.11137 (1992).

26. Yamamoto, D., Marmorini, G. \& Danshita, I. Quantum phase diagram of the triangular-lattice $x x z$ model in a magnetic field. Phys. Rev. Lett. 112, 127203. https://doi.org/10.1103/PhysRevLett.112.127203 (2014).

27. Starykh, O. A. Unusual ordered phases of highly frustrated magnets: A review. Rep. Prog. Phys. 78, 052502. https://doi.org/10. 1088/0034-4885/78/5/052502 (2015).

28. Quilliam, J. A. et al. Singlet ground state of the quantum antiferromagnet $\mathrm{Ba}_{3} \mathrm{CuSb}_{2} \mathrm{O}_{9}$. Phys. Rev. Lett. 109, 117203. https://doi. org/10.1103/PhysRevLett.109.117203 (2012).

29. Griset, C., Head, S., Alicea, J. \& Starykh, O. A. Deformed triangular lattice antiferromagnets in a magnetic field: Role of spatial anisotropy and dzyaloshinskii-moriya interactions. Phys. Rev. B 84, 245108. https://doi.org/10.1103/PhysRevB.84.245108 (2011).

30. Zhu, Z., Maksimov, P. A., White, S. R. \& Chernyshev, A. L. Disorder-induced mimicry of a spin liquid in $\mathrm{YbMgGaO}_{4}$. Phys. Rev. Lett. 119, 157201. https://doi.org/10.1103/PhysRevLett.119.157201 (2017).

31. Cantarino, M. R. et al. Dynamic magnetism in the disordered hexagonal double perovskite $\mathrm{BaTi}_{1 / 2} \mathrm{Mn}_{1 / 2} \mathrm{O}_{3}$. Phys. Rev. B 99, 054412. https://doi.org/10.1103/PhysRevB.99.054412 (2019).

32. Chanlert, P. et al. Field-driven successive phase transitions in the quasi-two-dimensional frustrated antiferromagnet $\mathrm{Ba}_{2} \mathrm{CoTeO}_{6}$ and highly degenerate classical ground states. Phys. Rev. B 93, 094420. https://doi.org/10.1103/PhysRevB.93.094420 (2016).

33. Chanlert, P., Kurita, N., Tanaka, H., Kimata, M. \& Nojiri, H. Collective and local excitations in $\mathrm{Ba}_{2} \mathrm{CoTeO}_{6}: \mathrm{A}$ composite system of a spin-1/2 triangular-lattice Heisenberg antiferromagnet and a honeycomb-lattice $J_{1}-J_{2}$ ising antiferromagnet. Phys. Rev. $B$ 96, 064419. https://doi.org/10.1103/PhysRevB.96.064419 (2017).

34. Ivanov, S. A., Nordblad, P., Mathieu, R., Tellgren, R. \& Ritter, C. Neutron diffraction studies and the magnetism of an ordered perovskite: $\mathrm{Ba}_{2} \mathrm{CoTeO}_{6}$. Dalton Trans. 39, 5490-5499. https://doi.org/10.1039/B927498G (2010).

35. Koga, T. et al. Magnetic structure of the $s=\frac{1}{2}$ quasi-two-dimensional square-lattice Heisenberg antiferromagnet $\mathrm{Sr}_{2} \mathrm{CuTeO}_{6}$. Phys. Rev. B 93, 054426. https://doi.org/10.1103/PhysRevB.93.054426 (2016).

36. Watanabe, M. et al. Valence-bond-glass state with a singlet gap in the spin- $-\frac{1}{2}$ square-lattice random $J_{1}-J_{2}$ Heisenberg antiferromagnet $\mathrm{Sr}_{2} \mathrm{CuTe}_{1-x} \mathrm{~W}_{x} \mathrm{O}_{6}$. Phys. Rev. B 98, 054422. https://doi.org/10.1103/PhysRevB.98.054422 (2018).

37. Mustonen, O. H. J. et al. Diamagnetic d-orbitals drive magnetic structure selection in the double perovskite ba2mnteo6. Chem. Mater. 32, 7070-7079. https://doi.org/10.1021/acs.chemmater.0c02971 (2020).

38. Li, L. et al. Magnetic ordering and spin dynamics in the $s=\frac{5}{2}$ staggered triangular lattice antiferromagnet $\mathrm{Ba}_{2} \mathrm{MnTeO}_{6}$. Phys. Rev. B 102, 094413. https://doi.org/10.1103/PhysRevB.102.094413 (2020).

39. Wulff, L., Wedel, B. \& Müller-Buschbaum, H. Zur kristallchemie von telluraten mit $\mathrm{Mn}^{2+}$ im kationischen und anionischen teil der kristallstruktur: $\left(\mathrm{Mn}_{2,4} \mathrm{Cu}_{0,6}\right) \mathrm{TeO}_{6}, \mathrm{Ba}_{2} \mathrm{MnTeO}_{6}$ und $\mathrm{Pb}\left(\mathrm{Mn}_{0,5} \mathrm{Te}_{0,5}\right) \mathrm{O}_{3} /$ on the crystal chemistry of tellurates containing $\mathrm{Mn}^{2+}$ in the cationic and anionic part of the crystal structure: $\left(\mathrm{Mn}_{2,4} \mathrm{Cu}_{0,6}\right) \mathrm{TeO}_{6}, \mathrm{Ba}_{2} \mathrm{MnTeO}_{6}$ and $\mathrm{Pb}\left(\mathrm{Mn}_{0,5} \mathrm{Te}_{0,5}\right) \mathrm{O}_{3}$. Z. Naturforsch. B 53, 49-52. https://doi.org/10.1515/znb-1998-0110 (1998).

40. Asai, S. et al. Magnetic ordering of the buckled honeycomb lattice antiferromagnet $\mathrm{Ba}_{2} \mathrm{NiTeO}_{6}$. Phys. Rev. B 93, 024412. https:// doi.org/10.1103/PhysRevB.93.024412 (2016).

41. Vasala, S. \& Karppinen, M. A ${ }_{2} B^{\prime} B^{\prime \prime} \mathrm{O}_{6}$ perovskites: A review. Prog. Solid. State Ch. 43, 1-36 (2015).

42. Momma, K. \& Izumi, F. Vesta: a three-dimensional visualization system for electronic and structural analysis. J. Appl. Cryst. 41, 653-658. https://doi.org/10.1107/S0021889808012016 (2008).

43. Toby, B. H. Expgui, a graphical user interface for gsas. J. Appl. Cryst. 34, 210-213. https://doi.org/10.1107/S0021889801002242 (2001).

44. Li, Y. et al. Gapless quantum spin liquid ground state in the two-dimensional spin-1/2 triangular antiferromagnet $\mathrm{YbMgGaO}_{4}$. Sci. Rep. 5, 16419. https://doi.org/10.1038/srep16419 (2015).

45. Li, Y. et al. Muon spin relaxation evidence for the U(1) quantum spin-liquid ground state in the triangular antiferromagnet $\mathrm{YbMg}$ $\mathrm{GaO}_{4}$. Phys. Rev. Lett. 117, 097201. https://doi.org/10.1103/PhysRevLett.117.097201 (2016).

46. Nath, R. et al. Magnetic transitions in the spin- $-\frac{5}{2}$ frustrated magnet $\mathrm{BiMn}_{2} \mathrm{PO}_{6}$ and strong lattice softening in $\mathrm{BiMn}_{2} \mathrm{PO}_{6}$ and $\mathrm{BiZn}_{2} \mathrm{PO}_{6}$ below 200 k. Phys. Rev. B 90, 024431. https://doi.org/10.1103/PhysRevB.90.024431 (2014). 
47. Tian, Z. et al. Susceptibility, high-field magnetization and esr studies in a spin-5/2 triangular-lattice antiferromagnet $\mathrm{Ba}_{3} \mathrm{MnSb}_{2}$ O. J. Magn. Magn. Mater. 360, 10-14. https://doi.org/10.1016/j.jmmm.2014.02.003 (2014).

48. Sugiyama, J. et al. Static magnetic order in metallic triangular antiferromagnet $\mathrm{Ag}_{2} \mathrm{MnO}_{2}$ detected by muon-spin spectroscopy. Phys. Rev. B 78, 104427. https://doi.org/10.1103/PhysRevB.78.104427 (2008).

49. Retuerto, M. et al. $\mathrm{Pb}_{2} \mathrm{MnTeO}_{6}$ double perovskite: An antipolar anti-ferromagnet. Inorg. Chem. 55, 4320-4329. https://doi.org/ 10.1021/acs.inorgchem.6b00054 (2016).

50. Ortega-San Martin, L. et al. Magnetic properties of the ordered double perovskite $\mathrm{Sr}_{2} \mathrm{MnTeO}_{6}$. Eur. J. Inorg. Chem. 2006, 13621370. https://doi.org/10.1002/ejic.200500880 (2006).

51. Robie, R. A. \& Hemingway, B. S. Low-temperature molar heat capacities and entropies of $\mathrm{MnO}_{2}$ (pyrolusite), $\mathrm{Mn}_{3} \mathrm{O}_{4}$ (hausmanite), and $\mathrm{Mn}_{2} \mathrm{O}_{3}$ (bixbyite). J. Chem. Thermodyn. 17, 165-181. https://doi.org/10.1016/0021-9614(85)90069-2 (1985).

52. Kittel, C. et al. Introduction to Solid State Physics Vol. 8 (Wiley, 1976).

53. Koteswararao, B. et al. Magnetic properties and heat capacity of the three-dimensional frustrated $s=\frac{1}{2}$ antiferromagnet $\mathrm{PbCuTe}_{2}$ $\mathrm{O}_{6}$. Phys. Rev. B 90, 035141. https://doi.org/10.1103/PhysRevB.90.035141 (2014).

54. Lashley, J. C. et al. Specific heat and magnetic susceptibility of the spinels $\mathrm{GeNi}_{2} \mathrm{O}_{4}$ and $\mathrm{GeCo}_{2} \mathrm{O}_{4}$. Phys. Rev. B 78, 104406. https:// doi.org/10.1103/PhysRevB.78.104406 (2008).

55. Hardy, V. et al. Phase transitions and magnetic structures in $\mathrm{MnW}_{1-x} \mathrm{Mo}_{x} \mathrm{O}_{4}$ compounds $(\mathrm{x} \leqslant 0.2)$. J. Phys. Condens. Matter. 28, 336003. https://doi.org/10.1088/0953-8984/28/33/336003 (2016).

56. Wolter, A. U. B. et al. Field-induced quantum criticality in the kitaev system $\alpha-\mathrm{RuCl}_{3}$. Phys. Rev. B 96, 041405. https://doi.org/10. 1103/PhysRevB.96.041405 (2017).

57. Janša, N. et al. Observation of two types of fractional excitation in the kitaev honeycomb magnet. Nat. Phys. 14, 786-790. https:// doi.org/10.1038/s41567-018-0129-5 (2018).

58. Ye, F. et al. Long-range magnetic interactions in the multiferroic antiferromagnet $\mathrm{MnWO}_{4}$. Phys. Rev. B 83, 140401. https://doi. org/10.1103/PhysRevB.83.140401 (2011).

59. Yaouanc, A. \& De Reotier, P. D. Muon Spin Rotation, Relaxation, and Resonance: Applications to Condensed Matter Vol. 147 (Oxford University Press, 2011).

60. Zorko, A. et al. Coexistence of magnetic order and persistent spin dynamics in a quantum kagome antiferromagnet with no intersite mixing. Phys. Rev. B 99, 214441. https://doi.org/10.1103/PhysRevB.99.214441 (2019).

61. Pregelj, M. et al. Persistent spin dynamics intrinsic to amplitude-modulated long-range magnetic order. Phys. Rev. Lett. 109, 227202. https://doi.org/10.1103/PhysRevLett.109.227202 (2012).

62. Yaouanc, A. et al. Evidence for unidimensional low-energy excitations as the origin of persistent spin dynamics in geometrically frustrated magnets. Phys. Rev. B 91, 104427. https://doi.org/10.1103/PhysRevB.91.104427 (2015).

63. Parida, P., Kashikar, R., Jena, A. \& Nanda, B. Universality in the electronic structure of $3 \mathrm{~d}$ transition metal oxides. J. Phys. Chem. Solids 123, 133-149. https://doi.org/10.1016/j.jpcs.2018.04.009 (2018).

64. Jena, A. \& Nanda, B. R. K. Unconventional magnetism and band gap formation in $\mathrm{LiFePO}_{4}$ : Consequence of polyanion induced non-planarity. Sci. Rep. 6, 19573. https://doi.org/10.1038/srep19573 (2016).

65. Noodleman, L. Valence bond description of antiferromagnetic coupling in transition metal dimers. J. Chem. Phys. 74, 5737-5743. https://doi.org/10.1063/1.440939 (1981).

66. Dai, D. et al. Analysis of the spin exchange interactions and the ordered magnetic structures of lithium transition metal phosphates $\mathrm{LiMPO}_{4}(\mathrm{M}=\mathrm{Mn}, \mathrm{Fe} \mathrm{Co}, \mathrm{Ni})$ with the olivine structure. Inorg. Chem. 44, 2407-2413 (2005).

67. Arh, T. et al. Origin of magnetic ordering in a structurally perfect quantum kagome antiferromagnet. Phys. Rev. Lett. 125, 027203. https://doi.org/10.1103/PhysRevLett.125.027203 (2020).

68. Zorko, A. et al. Magnetic inhomogeneity on a triangular lattice: the magnetic-exchange versus the elastic energy and the role of disorder. Sci. Rep. 5, 9272. https://doi.org/10.1038/srep09272 (2015).

69. Kawamura, H. Universality of phase transitions of frustrated antiferromagnets. J. Phys.:Condens. Matter 10, 4707-4754. https:// doi.org/10.1088/0953-8984/10/22/004 (1998).

70. Chowki, S., Kumar, R. \& Mohapatra, N. Long-range antiferromagnetic order and possible field induced spin-flop transition in $\mathrm{BiMnVO}_{5}$. J. Phys. Condens. Matter. 28, 486002. https://doi.org/10.1088/0953-8984/28/48/486002 (2016).

71. Ikedo, Y. et al. $\mu^{+}$SR study on triangular antiferromagnet $\mathrm{LiCrO}_{2}$. J. Phys. Conf. Ser. 225, 012016. https://doi.org/10.1088/17426596/225/1/012016 (2010).

72. Giannozzi, P. et al. Advanced capabilities for materials modelling with quantum espresso. J. Phys. Condens. Matter. $29,465901$. https://doi.org/10.1088/1361-648x/aa8f79 (2017).

73. Vanderbilt, D. Soft self-consistent pseudopotentials in a generalized eigenvalue formalism. Phys. Rev. B 41, 7892-7895. https:// doi.org/10.1103/PhysRevB.41.7892 (1990).

74. Perdew, J. P., Burke, K. \& Ernzerhof, M. Generalized gradient approximation made simple. Phys. Rev. Lett. 77, 3865-3868. https:// doi.org/10.1103/PhysRevLett.77.3865 (1996).

75. Cococcioni, M. \& de Gironcoli, S. Linear response approach to the calculation of the effective interaction parameters in the LDA + U method. Phys. Rev. B 71, 035105. https://doi.org/10.1103/PhysRevB.71.035105 (2005).

\section{Acknowledgements}

We thank DST, India for the PPMS facility at IIT Madras. PK acknowledges the funding by the Science and Engineering Research Board, and Department of Science and Technology, India through Research Grants. AZ acknowledges the funding by the Slovenian Research Agency through the Projects No. N1-0148, J1-2461, and Program No. P1-0125. BRKN acknowledges the funding by the Department of Science and Technology, India, through Grant No. CRG/2020/004330. BRKN acknowledges the use of the computing resources at HPCE, IIT Madras.HL acknowledges the financial support by SNF through the Project Grant 200021L-192109.

\section{Author contributions}

All authors contributed substantially to this work.

\section{Competing interests}

The authors declare no competing interests.

\section{Additional information}

Correspondence and requests for materials should be addressed to A.Z., B.R.K.N. or P.K.

Reprints and permissions information is available at www.nature.com/reprints. 
Publisher's note Springer Nature remains neutral with regard to jurisdictional claims in published maps and institutional affiliations.

(c) (1) Open Access This article is licensed under a Creative Commons Attribution 4.0 International License, which permits use, sharing, adaptation, distribution and reproduction in any medium or format, as long as you give appropriate credit to the original author(s) and the source, provide a link to the Creative Commons licence, and indicate if changes were made. The images or other third party material in this article are included in the article's Creative Commons licence, unless indicated otherwise in a credit line to the material. If material is not included in the article's Creative Commons licence and your intended use is not permitted by statutory regulation or exceeds the permitted use, you will need to obtain permission directly from the copyright holder. To view a copy of this licence, visit http://creativecommons.org/licenses/by/4.0/.

(C) The Author(s) 2021 